\title{
Comparison of high and low trans-fatty acid consumers: analyses of UK National Diet and Nutrition Surveys before and after product reformulation
}

\author{
Jayne Hutchinson ${ }^{1, *}$, Holly L Rippin ${ }^{1}$, Jo Jewell ${ }^{2}$, Joao J Breda ${ }^{2}$ and Janet E Cade ${ }^{1}$ \\ ${ }^{1}$ Nutritional Epidemiology Group (NEG), School of Food Science and Nutrition, University of Leeds, Leeds LS2 9JT, \\ UK: ${ }^{2}$ Division of Noncommunicable Diseases and Promoting Health through the Life-Course, WHO Regional Office \\ for Europe, Copenhagen, Denmark
}

Submitted 23 November 2016: Final revision received 24 August 2017: Accepted 30 August 2017: First published online 21 November 2017

\begin{abstract}
Objective: The WHO encourages the virtual elimination of artificial trans-fatty acids (TFA), which increase CHD risk. Our UK analysis explores whether voluntary reformulation results in differential TFA intakes among socio-economic groups by determining characteristics of high TFA consumers before and after product reformulation.

Design: Food intake was collected by $7 \mathrm{~d}$ weighed records pre-reformulation and $4 \mathrm{~d}$ diaries post-reformulation. Sociodemographic characteristics of TFA consumers above the WHO limit, and of the top $10 \%$ of TFA consumers as a percentage food energy, were compared with those of lower TFA consumers. Multivariate logistic regression determined independent socio-economic predictors of being a top $10 \%$ consumer.

Subjects: UK National Diet and Nutrition Surveys (NDNS) for adults aged 19-64 years pre-reformulation (2000/01; N 1724) and post-reformulation (2010/11-2011/ 12; $N$ 848).

Results: Post-reformulation $2.5 \%$ of adults exceeded the WHO limit, $v .57 \%$ prereformulation. In unadjusted analyses, high TFA consumption was associated with lower income, lower education and long-term illness/disability pre- but not postreformulation. In adjusted pre-reformulation analyses, degree holders were half as likely as those without qualifications to be top $10 \%$ consumers (OR $=0.51 ; 95 \% \mathrm{CI}$ $0.28,0.92)$. In adjusted post-reformulation analyses, those with higher income were $2 \cdot 5-3.3$ times more likely to be top $10 \%$ consumers than lowest income households. Pre-reformulation, high consumers consumed more foods containing artificial TFA, whereas ruminant TFA were more prominent post-reformulation. Conclusions: High TFA consumption was associated with socio-economic disadvantage pre-reformulation, but evidence of this is less clear postreformulation. Voluntary reformulation appeared effective in reducing TFA content in many UK products with mixed effects on dietary inequalities relating to income and education.
\end{abstract}

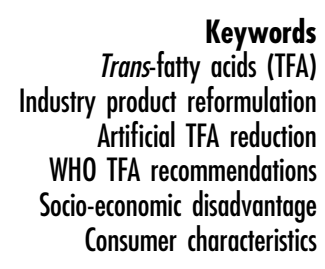

Trans-fatty acid (TFA) consumption is associated with increases in all-cause mortality ${ }^{(1)}$; for every $2 \%$ of total energy intake (\%TE) from TFA, CHD increases by $23 \%{ }^{(2)}$. There are two forms of TFA: one occurs naturally through biohydrogenation in the stomach of ruminant animals and the other is produced artificially in processed foods by hydrogenating vegetable or fish oils with hydrogen and a metal catalyst ${ }^{(3)}$. Although evidence of increased health risks from industrial trans-fatty acids (iTFA) is strong, a systematic review and meta-regression analysis reported that both industrial and ruminant TFA consumption were positively associated with reduced HDL, increased LDL and LDL:HDL cholesterol levels, which are directly associated with increased risk of $\mathrm{CVD}^{(4)}$.

Common iTFA sources include bakery products, processed meats, fat spreads, savoury snacks and fried fast foods. iTFA were added to processed foods to improve shelf-life, stability and palatability at a lower cost ${ }^{(5)}$. TFA removal has long been part of WHO global nutrition guidance $^{(6)}$ including the global action plan on prevention and control of non-communicable diseases ${ }^{(7)}$. The 2003 WHO/FAO technical report no. 916 stated that TFA intake 
should be minimised $(<1 \% \mathrm{TE})^{(8)}$. WHO called for a 'virtual elimination' from the food supply, based on evidence that setting legal TFA limits (typically $2 \mathrm{~g} / 100 \mathrm{~g}$ total fat) in food products is effective in meeting these intake goals $^{(9)}$. iTFA reduction has also been part of UK public health policy since 2011, including the Public Health Responsibility Deal, when the food industry confirmed ongoing efforts to remove iTFA from their products via a formal pledge ${ }^{(10)}$. Consequently, pressure on food manufacturers and retailers to reduce or remove iTFA has increased in recent years. Unlike some European countries which have introduced bans (including Austria, Denmark and Hungary) $)^{(11-14)}$, the UK has largely pursued TFA reduction via voluntary product reformulation e.g. by removing partially hydrogenated vegetable oils from foods and using advanced production techniques like modifying the fats and oils used in food preparation ${ }^{(10)}$.

Reducing iTFA intake could substantially reduce CHD mortality and health inequalities ${ }^{(15)}$. Modelling techniques indicate a $1 \%$ reduction of TFA in daily energy intake could result in 3900 fewer deaths and 37000 life-years gained, with the most deprived quintile benefitting the most. This modelling accounted for CHD mortality disproportionately affecting lower socio-economic groups ${ }^{(16)}$, but assumed equal TFA consumption. However, this reduction in deaths for the most deprived is likely to be underestimated because TFA may have been higher in this group ${ }^{(17)}$. The UK Low Income Diet and Nutrition Survey (LIDNS) 2003-2005 reported higher adult TFA intakes than the earlier 2000/01 UK nationally representative survey, both of which used pre-reformulation TFA values ${ }^{(17)}$. The National Diet and Nutrition Survey (NDNS) data collected in 2008-2012 ${ }^{(18)}$ show that average UK TFA intake meets the UK Dietary Reference Value of $<2 \%$ of food energy (\%FE) from TFA ${ }^{(19)}$ and the WHO recommended limit of $<1 \% \mathrm{TE}^{(8)}$. However, these averages potentially mask problems associated with higher intakes in certain groups ${ }^{(20)}$.

Previous reviews have suggested that, globally, voluntary measures may be less effective than legislation in reducing TFA and intake inequalities ${ }^{(21)}$. In part, this may be due to difficulties ensuring the participation of a critical mass of manufacturers and retailers, especially small and medium-sized enterprises, which dominate the food sector ${ }^{(22)}$. In New York State some counties imposed iTFA limits of $0.5 \mathrm{~g} /$ portion in food-service establishments, resulting in an estimated $4.5 \%$ reduction in CVD-related deaths ${ }^{(23)}$. Elsewhere, a ban has been favoured to maximise impact for all socio-economic groups and create a level playing field for companies. For example, CVD mortality in Denmark fell more than expected following a ban on TFA above $2 \mathrm{~g} / 100 \mathrm{~g}$ fats/oils in $2003^{(24)}$. The UK experience with TFA reformulation is therefore a good case study to explore the potential impact, strengths and limitations of voluntary reformulation ${ }^{(22)}$.

Our research analysed pre-reformulation and postreformulation TFA data from the UK NDNS to determine characteristics of high TFA consumers compared with lower consumers at these different time points, highlighting the potential impact of reformulation with particular reference to socially disadvantaged groups.

\section{Methods}

NDNS dietary and sociodemographic data were analysed and compared from surveys representing before (2000/01) and after (2010-2012) TFA product reformulation. The prereformulation analysis used NDNS data for 1724 UK adults aged 19-64 years collected in 2000/01 ${ }^{(25)}$. The separate post-reformulation analysis used data which incorporated the reduced TFA content of reformulated products for 848 adults (restricted to ages 19-64 years) from Years 3 and 4 (2010/11 and 2011/12) of the 2008-2012 NDNS Rolling Programme $(\mathrm{RP})^{(18)}$. Years 1 and 2 of the NDNS RP were not included in the analyses because NDNS RP Year 1 data did not incorporate post-reformulation TFA compositions and Year 2 data incorporated only some changes. The samples were drawn from the GB and UK Postcode Address Files, selected using multistage random probability sampling with postal sectors as the primary sampling units. All food and nutrient variables were derived from shortterm food records or diaries and sociodemographic variables were derived from NDNS questionnaire responses collected alongside food intake.

The NDNS 2000/01 collected food data using $7 \mathrm{~d}$ weighed intake dietary records for all foods and drinks consumed $^{(25)}$. The NDNS 2008-2012 RP used a $4 \mathrm{~d}$ consecutive food diary and portion sizes were estimated using household measures and food packaging labels ${ }^{(18)}$. TFA values in the composition databanks underpinning the NDNS 2000/01 survey were based mainly on food composition analyses carried out in the 1990s, using composite samples of various brands of similar foods. When no analytical data for a food were available, the TFA value was estimated using manufacturer/retailer data for total and saturated fat (typically from the product label) and the fatty acid profile of similar foods ${ }^{(26)}$. Updates on laboratoryanalysed TFA levels in processed foods high in iTFA which had been targeted for reformulation were reported in 2011 and $2013^{(27,28)}$. These included biscuits, buns, cakes, pastries and products bought in 2008 and reported in $2011^{(27)}$. The 2013 report included pizza, garlic bread, breakfast cereal, quiche, fat spreads, cooking fats and oils, chicken products, meat pies, fish products, chips, coleslaw, crisps and savoury snacks, confectionary, chocolate spread, soup, baby rusks and ice cream ${ }^{(28)}$. The UK Department of Health incorporated these values into the nutrient databanks supporting Years 3 and 4 (2010/11 and 2011/12) of the NDNS RP. Forty-three per cent of the 2900 products (which included naturally occurring TFA) in the Year 1 nutrient databanks, with non-zero TFA values in Year 1 and Year 4, had updated TFA values in the Year 4 nutrient databank. 
Only the main product groups for milk, fruit, salads and raw vegetables, drinks and supplements did not have TFA value updates. The products analysed for the update were purchased between 2008 and 2010 and were mostly popular and widely purchased products in the $\mathrm{UK}^{(27,28)}$. Sub-samples of food products had been combined in equal weights to form a composite sample for analysis, with five to sixteen sub-samples for each category ${ }^{(28)}$. The UK Department of Health incorporated the new TFA values into the new composition tables.

\section{Statistical methods}

Two methods of grouping NDNS individuals were used to compare the characteristics of high and lower TFA consumers:

1. individuals who consumed over the current $\mathrm{WHO}$ recommended limit on $\mathrm{TFA}^{(8)}$, i.e. $\geq 1 \% \mathrm{TE}$ from TFA, compared with those who consumed $<1 \% \mathrm{TE}$ from TFA; and

2. the top $10 \%$ of TFA consumers in terms of percentage of energy from food intake compared with the remaining $90 \%$.

The second analysis was undertaken because it provided more power to find associations between high TFA consumption and sociodemographic variables. It also excludes alcohol from energy intake, which may dilute findings. For each analysis, the following sociodemographic characteristics were compared for adults: (i) age, continuous and grouped (19-34, 35-49, 50-64 years); (ii) gender; (iii) qualifications (no qualifications; school certificates and other qualifications; higher education below degree; degree); (iv) in employment (i.e. economically active or in full-time education, yes/no); (v) gross income (split into five groups); (vi) social class (NDNS 2000/01 used the Registrar General's Standard Occupational Classification and NDNS 2010-2012 used the National Statistics Socio-economic Classification); (vii) region (Northern England; Midlands; London, East and South England; Scotland, Wales (and Northern Ireland for NDNS RP Years 3 and 4)); (viii) number of adults in household (aged over 16 years); (ix) child in household (yes/no); (x) marital status (single/never married; married and living together/cohabiting; separated/divorced/ widowed); (xi) White or non-White; (xii) ethnic group (White, Black or Black British, Asian or Asian British, Other group); and (xiii) longstanding illness or disability (yes/no). NDNS income data were provided in income bands and not as a continuous variable; we collapsed these into five bands (see notes to tables) so that the nationally representative weighted percentages in the respective bands were similar in both surveys. Additionally, for the NDNS 2010-2012 analyses the following comparisons were undertaken: (xiv) Index of Multiple Deprivation (IMD) by quintile; and (xv) equivalised household income by continuous variable and quintile.
Potential associations between high TFA and alcohol intake were assessed using alcohol intake data on the day of highest alcohol consumption in the seven days (none, within daily recommended levels, between recommended and binge drinking levels, binge drinking levels; results not shown in tables).

In these univariate analyses, the means of continuous data were compared using $t$ tests and categorical data were compared using $\chi^{2}$ tests. Mean intakes for selected nutrients that were reasonably normally distributed were compared by $t$ tests between the high and lower TFA groups. Macronutrients: TFA ( $\mathrm{g}, \% \mathrm{TE}, \% \mathrm{FE})$; total energy (kJ/kcal); food energy (kJ/kcal); fat (\%FE); saturated fat (\%FE); non-milk extrinsic sugars (\%FE). Micronutrients per 4184 kJ (1000 kcal): $\mathrm{Na}(\mathrm{mg})$; vitamin $\mathrm{C}(\mathrm{mg})$; vitamin D $(\mu \mathrm{g})$; vitamin $\mathrm{E}$ (mg). Additionally, the individual percentage contribution of all main food and beverage groups (approximately sixty) to total food energy intake and total TFA intake were analysed; those contributing less than $0.5 \%$ were not tabled.

Multivariate logistic regression analyses of the NDNS data sets were undertaken to determine which sociodemographic characteristics were independently associated with high TFA consumption over other characteristics. Due to low numbers in the post-reformulation surveys consuming above the WHO recommended TFA limit and the potential distortion of total energy intake by high alcohol consumers, multivariate analyses were conducted on the top $10 \%$ of TFA consumers as a percentage of food energy intake (rather than total energy intake). For the multivariate regression analyses, we included the following variables common to both the pre- and post-reformulation NDNS surveys: food energy intake per $418 \mathrm{~kJ}$ (100 kcal); age (continuous); gender; number of adults in household (continuous); number of children in household (continuous); and (as categorised above): qualification, income, region, marital status, ethnicity and longstanding illness. The variable for IMD quintiles was included in sensitivity analyses for the recent NDNS RP; however, this information was not available for analyses on the earlier NDNS 2000/01 data set.

Within- and between-person variations in TFA intake were calculated to produce a predicted 'usual' intake distribution and to re-estimate the proportion above the WHO recommended limit for both pre- and postreformulation surveys. The common framework method with square-root-transformed intakes was used, as described in Dodd et al. ${ }^{(29)}$. Because no individual in the recent NDNS (2010-2012) survey had TFA intake over the WHO recommendation using usual intake calculations, it was not possible to create new TFA consumer groups with this method and repeat the comparative analyses described above. Additionally, because the usual intake model shrinks all intakes towards the mean, the same individuals would be in the top $10 \%$ of the usual intake distribution as found in the original top $10 \%$ of TFA consumers; therefore, additional tables for this could not be produced. 
The response rate for completing a $7 \mathrm{~d}$ diary in the NDNS 2000/01 was $47 \%$ of the eligible sample; and for the NDNS RP was 53\% for Year 3 and 55\% for Year 4 recording dietary intake. Analyses were weighted using the NDNS survey weights provided, to produce estimated results representative of the UK population, accounting for non-responses ${ }^{(30,31)}$. Statistical significance was set at $P<0.05$. Analyses used the Stata statistical software package versions 13 and 14 .

\section{Results}

The age ranges in the nationally representative weighted pre- and post-reformulation samples were similar, being 37,33 and $30 \%$ for the age groups of 19-34, 35-49 and 50-64 years, respectively, in the UK NDNS 2000/01, and 35, 36 and 29\%, respectively, in Years 3 and 4 (2010/11 and 2011/12) of the recent UK NDNS RP. The proportion of males (49 and 48\%) was also similar. There were differences regarding ethnicity and degree attainment: $94 \%$ were White and 19\% held a degree in the prereformulation surveys, whereas $85 \%$ were White and $28 \%$ held a degree in the post-reformulation surveys.

In the UK NDNS 2000/01, on average men and women aged 19-64 years consumed $1.1 \%$ of their total and $1.2 \%$ of their food energy from TFA. In these pre-reformulation analyses, $57 \%$ of adults ( $57 \%$ of males; $58 \%$ of females) consumed over the current WHO recommended TFA limit, i.e. $\geq 1 \%$ TE from TFA. The average consumption of those consuming over the WHO limit was $1.4 \%$ of total and food energy intake. In terms of the UK TFA recommendations, only $4 \%$ of men and women consumed $\geq 2 \% \mathrm{FE}$ from TFA.

In Years 3 and 4 (2010/11 and 2011/12) of the recent UK NDNS RP, on average adults aged 19-64 years consumed $0.51 \%$ of their total $(0.50 \%$ for men; $0.52 \%$ for women) and $0.53 \%$ of their food energy ( $0.53 \%$ for men; $0.54 \%$ for women) from TFA. On average, $2.5 \%$ of adults consumed over the current WHO TFA limit $(1.9 \%$ of males; $3.0 \%$ of females). The average TFA intake of those consuming above the WHO limit was $1.2 \% \mathrm{TE}$ and $1.3 \%$ FE. In terms of the UK TFA recommendation, only one woman $(<0.01 \%)$ and no men $(0 \%)$ aged $19-64$ years in this survey consumed $\geq 2 \% \mathrm{FE}$ from TFA.

\section{Sociodemographic characteristics of higher consumers of trans-fatty acids}

In the UK NDNS 2000/01 unadjusted analyses, adults consuming over the WHO recommended TFA limit were significantly more likely to have middle to lower incomes, no qualifications and be White than those below the WHO TFA limit (Table 1). No significant differences were found regarding other sociodemographic data. Conversely, in the recent UK NDNS (2010/11 and 2011/12), individuals consuming over the WHO TFA limit did not have significantly different sociodemographic characteristics compared with those who consumed less (Table 1). Although a higher proportion of White individuals and those with no qualifications consumed over the WHO TFA recommendation post-reformulation, the difference was not significant. In relation to alcohol intake, individuals consuming below the WHO TFA limit were more likely to be binge drinkers than those consuming TFA above recommendations pre-reformulation $(38.5$ v. $24.6 \%$, $P<0 \cdot 001$ ), but not post-reformulation.

In the NDNS 2000/01 unadjusted analyses, some sociodemographic characteristics were associated with being in the top $10 \%$ of TFA consumers in terms of percentage of food energy (Table 2). As found for those consuming above the WHO recommended TFA limit, the top $10 \%$ consumers were more likely to have middle to lower incomes, no qualifications and longstanding illnesses. Regional differences were also found, but there were no differences regarding ethnicity. In the recent NDNS RP (2010/11 and 2011/12), in contrast to the NDNS $2000 / 01$, income was not associated with being in the top $10 \%$ of TFA consumers (Table 2). The top $10 \%$ of TFA consumers were more likely than the remaining $90 \%$ to have no qualifications and reside in the most deprived areas of England.

In the multivariate logistic regression analyses of the top $10 \%$ of adult TFA consumers in the pre-reformulation NDNS 2000/01, there were significant differences relating to education and region (Table 3 ). Adults with a degree were half as likely to be top $10 \%$ TFA consumers as those with no qualifications (adjusted $\mathrm{OR}=0.51 ; 95 \%$ CI $0 \cdot 28$, 0.92 ; Table 3). Individuals living in the North of England (adjusted $\mathrm{OR}=0.53 ; 95 \% \mathrm{CI} 0.33,0.85$ ), Scotland and Wales (adjusted OR $=0.33$; 95\% CI 0.19, 0.60) were less likely to be in the top $10 \%$ of TFA consumers than adults in living in London, East and South of England. However, unlike the unadjusted analyses, there was no evidence of significant differences relating to income and long-term illness in the multivariate analyses. In contrast, multivariate logistic regression analyses of the recent NDNS RP showed income-related differences as significant. Higher income groups were $2 \cdot 5-3 \cdot 25$ times more likely to be top $10 \%$ TFA consumers than those in the lowest income group; those with household income of $£ 2000-30000$ were most likely to be high TFA consumers than households with income below £15000 (adjusted OR=3.25; 95\% CI 1.10, 9.62). Regional differences also became significant in multivariate analyses of the NDNS RP, but unlike in the earlier NDNS 2000/01, the top $10 \%$ of consumers were more likely to reside in the Midlands than London, East and South England (adjusted OR $=2 \cdot 40 ; 95 \%$ CI $1 \cdot 13$, 5.11). In sensitivity analyses, inclusion of the IMD variable quintiles into the NDNS RP multivariate analyses did not change most of the results substantially. However, being a top $10 \%$ TFA consumer was about $70 \%$ less likely for those with higher education below degree compared with those with no education (adjusted OR $=0 \cdot 25 ; 95 \%$ CI 0.07, 0.97) 
Table 1 Sociodemographic characteristics of adults (aged 19-64 years) in the National Diet and Nutrition Survey (NDNS 2000/01) and in Years 3 and 4 of the National Diet and Nutrition Survey Rolling Programme (NDNS RP 2010/11 and 2011/12) who consumed $1 \%$ or more of their total energy intake from trans-fatty acids (TFA) compared with those who consumed less

\begin{tabular}{|c|c|c|c|c|c|c|c|c|}
\hline \multirow[b]{3}{*}{ Adults: WHO TFA limit } & \multicolumn{3}{|c|}{ NDNS 2000/01 } & \multirow[b]{3}{*}{$P$ value } & \multicolumn{4}{|c|}{ Years $3 \& 4$ of NDNS RP 2010/11 and 2011/12 } \\
\hline & $\begin{array}{c}\text { Total } \\
(N 1724, \\
\text { weighted } \\
N 1724)\end{array}$ & $\begin{array}{c}\geq 1 \% \text { TE } \\
\text { from TFA } \\
(N 988, \\
\text { weighted } \\
N 988)\end{array}$ & $\begin{array}{c}<1 \% \text { TE } \\
\text { from TFA } \\
(N 736, \\
\text { weighted } \\
N 736)\end{array}$ & & $\begin{array}{c}\text { Total } \\
\text { (N 848, } \\
\text { weighted } \\
N \text { 1277) }\end{array}$ & $\begin{array}{c}\geq 1 \% \text { TE } \\
\text { from TFA } \\
(N 22, \\
\text { weighted } \\
N 32)\end{array}$ & $\begin{array}{c}<1 \% \text { TE } \\
\text { from TFA } \\
(N 826, \\
\text { weighted } \\
N 1245)\end{array}$ & \\
\hline & Unweighted $n$ & $\%^{*}$ & $\%^{*}$ & & Unweighted $n$ & $\% *$ & $\%^{*}$ & $P$ value \\
\hline TFA $(g)$, mean & & $3 \cdot 11$ & 1.59 & $<0.001$ & & $2 \cdot 18$ & 1.02 & $<0.001$ \\
\hline SD & & 1.30 & 0.66 & & & 0.62 & 0.53 & \\
\hline Age (years), mean & & $40 \cdot 96$ & 40.04 & 0.2 & & 40.94 & 41.30 & 0.9 \\
\hline SD & & $12 \cdot 85$ & $12 \cdot 50$ & & & 14.83 & $12 \cdot 79$ & \\
\hline Age group & & & & 0.6 & & & & 0.9 \\
\hline 19-34 years & 510 & $36 \cdot 8$ & 37.9 & & 245 & 33.9 & 34.6 & \\
\hline $35-49$ years & 682 & $32 \cdot 6$ & 33.7 & & 330 & 33.3 & $36 \cdot 3$ & \\
\hline 50-64 years & 532 & $30 \cdot 6$ & $28 \cdot 4$ & & 273 & $32 \cdot 8$ & $29 \cdot 1$ & \\
\hline Males & 766 & 48.0 & 48.8 & 0.8 & 484 & 38.5 & 49.5 & 0.4 \\
\hline Qualifications & & & & 0.03 & & & & 0.5 \\
\hline $\begin{array}{l}\text { No qualifications } \\
\text { (or in full-time education) }\end{array}$ & 301 & $18 \cdot 1$ & $13 \cdot 3$ & & 138 & $18 \cdot 2$ & $16 \cdot 7$ & \\
\hline $\begin{array}{l}\text { School certificates \& other } \\
\text { qualifications }\end{array}$ & 856 & $51 \cdot 3$ & $51 \cdot 3$ & & 374 & $51 \cdot 2$ & 43.9 & \\
\hline Higher education below degree & 245 & $13 \cdot 3$ & 14.6 & & 97 & 0.00 & $11 \cdot 2$ & \\
\hline Degree & 321 & $17 \cdot 2$ & 20.8 & & 237 & 30.5 & $28 \cdot 2$ & \\
\hline In employment & 1280 & $77 \cdot 8$ & $75 \cdot 8$ & 0.3 & 609 & $82 \cdot 5$ & $73 \cdot 1$ & 0.3 \\
\hline Gross household income† & & & & $<0.001$ & & & & 0.7 \\
\hline Lowest income group & 243 & $10 \cdot 6$ & 11.0 & & 123 & $14 \cdot 2$ & $12 \cdot 9$ & \\
\hline 2 & 267 & $15 \cdot 0$ & $10 \cdot 3$ & & 141 & 10.5 & $19 \cdot 1$ & \\
\hline 3 & 253 & $17 \cdot 4$ & 11.8 & & 85 & $18 \cdot 1$ & $10 \cdot 3$ & \\
\hline 4 & 381 & $24 \cdot 1$ & 24.5 & & 141 & $15 \cdot 9$ & $20 \cdot 3$ & \\
\hline Highest income group & 548 & 32.9 & $42 \cdot 4$ & & 239 & $41 \cdot 3$ & 37.4 & \\
\hline $\begin{array}{l}\text { Equalised household income } \\
(£) \text {, mean }\end{array}$ & NA & NA & NA & & 729 & 37798 & 32874 & 0.5 \\
\hline SD & & & & & & 27512 & 23885 & \\
\hline Equivalised household income & & & & & & & & 0.9 \\
\hline Lowest income quintile & NA & NA & NA & & 129 & $14 \cdot 2$ & $17 \cdot 3$ & \\
\hline 2 & & & & & 114 & 13.6 & $16 \cdot 4$ & \\
\hline 3 & & & & & 124 & 11.5 & $19 \cdot 1$ & \\
\hline 4 & & & & & 170 & 29.0 & $22 \cdot 0$ & \\
\hline Highest income quintile & & & & & 192 & 31.6 & $25 \cdot 2$ & \\
\hline $\begin{array}{l}\text { Quintile of IMD score, England } \\
\text { only }\end{array}$ & & & & & & & & 0.7 \\
\hline Most deprived & NA & NA & NA & & 131 & 9.8 & $15 \cdot 5$ & \\
\hline 2 & & & & & 142 & 9.2 & $18 \cdot 4$ & \\
\hline 3 & & & & & 157 & $21 \cdot 6$ & $18 \cdot 7$ & \\
\hline 4 & & & & & 138 & 14.0 & $16 \cdot 8$ & \\
\hline Least deprived & & & & & 133 & $22 \cdot 3$ & $15 \cdot 8$ & \\
\hline $\begin{array}{l}\text { Other UK countries, not } \\
\text { England }\end{array}$ & & & & & 147 & $23 \cdot 1$ & $15 \cdot 5$ & \\
\hline Socio-economic classification $\ddagger$ & & & & 0.8 & & & & 0.9 \\
\hline Highest & 100 & 6.5 & 5.4 & & 361 & $46 \cdot 2$ & 44.4 & \\
\hline & 528 & 27.7 & 33.8 & & 170 & $16 \cdot 9$ & 20.5 & \\
\hline & 401 & 23.5 & $25 \cdot 3$ & & 300 & $36 \cdot 9$ & $35 \cdot 2$ & \\
\hline & 282 & $18 \cdot 8$ & $16 \cdot 1$ & & & & & \\
\hline & 283 & $17 \cdot 0$ & 14.7 & & & & & \\
\hline Lowest & 98 & 6.5 & 4.8 & & & & & \\
\hline Region & & & & 0.6 & & & & 0.7 \\
\hline Northern England & 451 & $25 \cdot 8$ & $28 \cdot 3$ & & 201 & $28 \cdot 8$ & $23 \cdot 6$ & \\
\hline Midlands & 266 & $16 \cdot 3$ & $14 \cdot 3$ & & 148 & $16 \cdot 3$ & $15 \cdot 5$ & \\
\hline $\begin{array}{l}\text { London, East \& South } \\
\text { England }\end{array}$ & 808 & $46 \cdot 0$ & $45 \cdot 0$ & & 352 & $32 \cdot 5$ & $45 \cdot 1$ & \\
\hline $\begin{array}{l}\text { Scotland, Wales } \\
(+\mathrm{NI} \text { in NDNS RP) }\end{array}$ & 199 & $11 \cdot 9$ & $12 \cdot 3$ & & 147 & $22 \cdot 3$ & $15 \cdot 8$ & \\
\hline $\begin{array}{l}\text { Number of adults in household, } \\
\text { mean }\end{array}$ & & $2 \cdot 28$ & $2 \cdot 22$ & 0.3 & & $2 \cdot 37$ & $2 \cdot 30$ & 0.8 \\
\hline SD & & 0.93 & 0.90 & & & 0.98 & 0.94 & \\
\hline Child in household, yes & 644 & $36 \cdot 1$ & 38.0 & 0.4 & 328 & 49.8 & $39 \cdot 4$ & 0.4 \\
\hline
\end{tabular}




\begin{tabular}{|c|c|c|c|c|c|c|c|c|}
\hline \multirow[b]{3}{*}{ Adults: WHO TFA limit } & \multicolumn{3}{|c|}{ NDNS 2000/01 } & & \multicolumn{4}{|c|}{ Years $3 \& 4$ of NDNS RP $2010 / 11$ and $2011 / 12$} \\
\hline & $\begin{array}{c}\text { Total } \\
(N 1724, \\
\text { weighted } \\
N 1724)\end{array}$ & $\begin{array}{c}\geq 1 \% \text { TE } \\
\text { from TFA } \\
(N 988, \\
\text { weighted } \\
N 988)\end{array}$ & $\begin{array}{c}<1 \% \text { TE } \\
\text { from TFA } \\
(N 736, \\
\text { weighted } \\
N 736)\end{array}$ & & $\begin{array}{c}\text { Total } \\
(N \text { 848, } \\
\text { weighted } \\
N \text { 1277) }\end{array}$ & $\begin{array}{c}\geq 1 \% \text { TE } \\
\text { from TFA } \\
(N 22, \\
\text { weighted } \\
N 32)\end{array}$ & $\begin{array}{c}<1 \% \text { TE } \\
\text { from TFA } \\
(N 826, \\
\text { weighted } \\
N 1245)\end{array}$ & \\
\hline & Unweighted $n$ & $\% *$ & $\%^{*}$ & $P$ value & Unweighted $n$ & $\% *$ & $\% *$ & $P$ value \\
\hline Marital status & & & & 0.8 & & & & 0.2 \\
\hline Single/never married & 358 & $19 \cdot 7$ & $21 \cdot 1$ & & 188 & $34 \cdot 8$ & $20 \cdot 1$ & \\
\hline $\begin{array}{l}\text { Married/living together/ } \\
\text { cohabiting }\end{array}$ & 1075 & $70 \cdot 6$ & $69 \cdot 0$ & & 530 & $54 \cdot 2$ & $70 \cdot 9$ & \\
\hline $\begin{array}{l}\text { Separated/divorced/ } \\
\text { widowed }\end{array}$ & 291 & $9 \cdot 8$ & 9.9 & & 130 & $11 \cdot 0$ & 9.0 & \\
\hline Ethnic group, two categories & & & & $<0.001$ & & & & 0.2 \\
\hline White & 1629 & $95 \cdot 6$ & $91 \cdot 7$ & & 750 & 93.1 & $85 \cdot 0$ & \\
\hline Non-White & 95 & 4.4 & 8.3 & & 98 & 6.9 & $15 \cdot 0$ & \\
\hline Ethnic group & & & & 0.001 & & & & 0.8 \\
\hline White & 1629 & $95 \cdot 6$ & $91 \cdot 7$ & & 750 & 93.1 & $85 \cdot 0$ & \\
\hline Black or Black British & 29 & 0.9 & 2.5 & & 29 & $4 \cdot 1$ & 4.0 & \\
\hline Asian or Asian British & 35 & $2 \cdot 1$ & 3.2 & & 40 & 2.9 & 6.5 & \\
\hline Any Other group incl. mixed & 31 & 1.4 & $2 \cdot \overline{6}$ & & 29 & 0.0 & 4.5 & \\
\hline Has longstanding illness, yes & 655 & 37.9 & 34.9 & 0.3 & 292 & 30.7 & $31 \cdot 3$ & 1.0 \\
\hline
\end{tabular}

$\%$ TE, percentage of total energy; NI, Northern Ireland; NA, not available; TFA intake $\geq 1 \%$ TE is above the WHO recommended TFA limit.

*Values presented are percentages, unless indicated otherwise.

†Gross annual household income in the previous 12 months groupings for NDNS 2000/01 are: $<£ 8000, £ 8000$ to $<£ 12000, £ 12000$ to $<£ 18000$, £18000 to $<£ 25000, \geq £ 25000$; for NDNS RP are: $<£ 15000, £ 15000$ to $<£ 20000, £ 20000$ to $<£ 30000, £ 30000$ to $<£ 40000, \geq £ 40000$.

¥Social class groups for NDNS 2000/01 are: I, professional; II, managerial and technical; IIIN, skilled non-manual; IIIM, skilled manual; IV, semi-skilled; V, unskilled; socio-economic classification grouping for NDNS RP are: Managerial \& Professional, Intermediate \& Small businesses, Routine \& Never worked.

and for those in the second most deprived group compared with the most deprived (adjusted OR $=0 \cdot 27 ; 95 \% \mathrm{CI}$ $0 \cdot 09,0 \cdot 82$ ).

\section{Foods contributing most to trans-fatty acid intake in higher trans-fatty acid consumers}

Pre-reformulation, $76 \%$ of TFA intake in those over the WHO limit was spread across meat (20\%), dairy (20\%), biscuits, buns, cakes, pastries, fruit pies and puddings (21\%) and spreads (15\%; Table 4). For those meeting the recommendation, the largest percentage (50\%) came from meat and dairy. In contrast, post-reformulation, $78 \%$ of TFA intake of those over the WHO limit came from dairy (46\%) and meat (32\%), with cheese, butter, cream, lamb, burgers and kebabs being the highest contributors totalling $62 \%$. Only $12 \%$ was contributed by biscuits, buns, cakes, pastries, fruit pies and puddings and $<1 \%$ by spreads (Table 4 ). For those meeting the WHO recommendation, smaller proportions of TFA intake came from dairy products (41\%) and meat (26\%), with only $6 \%$ from biscuits, buns, cakes, pastries, fruit pies and puddings.

Regarding the top $10 \%$ of TFA consumers pre-reformulation, $20 \%$ of TFA came from meat, $14 \%$ from dairy, $23 \%$ from spreads and $24 \%$ from biscuits, buns, cakes, pastries, fruit pies and puddings (Table 5). Biscuits, reduced-fat spreads and meat pies and pasties contributed $38 \%$ of TFA intake, which was significantly higher than in the remaining $90 \%$. In contrast, post-reformulation, $70 \%$ of TFA for the top $10 \%$ of TFA consumers came from just dairy (46\%) and meat (24\%), and only $8 \%$ from biscuits, buns, cakes, pasties, fruit pies and puddings, and $1 \%$ from spreads (other than butter); similar percentages were found for the remaining $90 \%$ of lower TFA consumers (Table 5). Of note, TFA intakes from butter, cream and lamb were significantly higher for the top $10 \%$ of TFA consumers, and TFA intakes from semi-skimmed milk and beef significantly lower (Table 5). Additionally, the percentage of energy from food groups appeared to have a similar distribution in the pre- and post-reformulation surveys (Tables 4 and 5).

\section{Nutritional intake of higher consumers of trans- fatty acids}

Adults in the NDNS 2000/01 who consumed above the WHO TFA limit had significantly higher energy and fat intakes (absolute and as \%FE), lower $\mathrm{Na}$, vitamins $\mathrm{C}, \mathrm{D}$ and $\mathrm{E}$ intakes, and lower total fruit and vegetable and vitamin $\mathrm{C}$ intakes per $4184 \mathrm{~kJ}$ (1000 kcal; Table 6). Similar differences were also found in analyses of the top $10 \%$ of TFA consumers as a percentage of food energy, apart from $\mathrm{Na}$ and vitamin $\mathrm{D}$, which were not significant (Table 7 ).

In the analyses of the recent NDNS (2010/11 and 2011/ 12), high TFA consumers had higher total fat, saturated fat and lower vitamin E intakes (Tables 6 and 7). Those consuming above the WHO recommendation also had lower intakes of total energy and vitamin D per $4184 \mathrm{~kJ}$ (1000 kcal; Table 6). The top $10 \%$ of TFA consumers also had lower non-milk extrinsic sugars, vitamin $\mathrm{C}$, and fruit and vegetable intakes. 
Table 2 Sociodemographic characteristics of adults (aged 19-64 years) in the National Diet and Nutrition Survey (NDNS 2000/01) and Years 3 and 4 of the National Diet and Nutrition Survey Rolling Programme (NDNS RP 2010/11 and 2011/12) who were the top 10\% of trans-fatty acid (TFA) consumers as a percentage of food energy compared with the remaining $90 \%$

\begin{tabular}{|c|c|c|c|c|c|c|c|c|}
\hline \multirow{3}{*}{$\begin{array}{l}\text { Adults: top } 10 \% \text { TFA } \\
\text { consumers }\end{array}$} & \multicolumn{3}{|c|}{ NDNS 2000/01 } & \multicolumn{5}{|c|}{ NDNS RP 2010/11 and 2011/12 } \\
\hline & $\begin{array}{c}\text { Total } \\
(N 1724, \\
\text { weighted } \\
N 1724)\end{array}$ & $\begin{array}{c}\text { Top } 10 \% \\
\text { TFA } \\
\text { consumers } \\
\text { as } \% F E \\
(N 191, \\
\text { weighted } \\
N 176)\end{array}$ & $\begin{array}{c}\text { Remaining } \\
90 \% \\
(N 1533 \\
\text { weighted } \\
N 1548)\end{array}$ & & $\begin{array}{c}\text { Total } \\
(N 848, \\
\text { weighted } \\
N 1277)\end{array}$ & $\begin{array}{c}\text { Top } 10 \% \\
\text { TFA } \\
\text { consumers } \\
\text { as \%FE } \\
(N 88, \\
\text { weighted } \\
N 130)\end{array}$ & $\begin{array}{c}\text { Remaining } \\
90 \% \\
(N 760 \\
\text { weighted } \\
N 1147)\end{array}$ & \\
\hline & Unweighted $n$ & $\%^{*}$ & $\%^{*}$ & $P$ value & Unweighted $n$ & $\%^{*}$ & $\%^{*}$ & $P$ value \\
\hline TFA intake (g), mean & & 4.55 & $2 \cdot 22$ & $<0.001$ & & 1.93 & 0.95 & $<0.001$ \\
\hline SD & & 1.00 & 1.85 & & & 0.59 & 0.46 & \\
\hline Age (years), mean & & 41.94 & $40 \cdot 41$ & 0.2 & & $42 \cdot 90$ & $41 \cdot 10$ & 0.3 \\
\hline SD & & 12.95 & $12 \cdot 68$ & & & $13 \cdot 12$ & $12 \cdot 80$ & \\
\hline Age group & & & & 0.3 & & & & 0.5 \\
\hline $19-34$ years & 510 & 31.7 & 37.9 & & 245 & $29 \cdot 0$ & $35 \cdot 2$ & \\
\hline $35-49$ years & 682 & 33.9 & 33.0 & & 330 & $36 \cdot 9$ & $36 \cdot 2$ & \\
\hline 50-64 years & 532 & 34.4 & $29 \cdot 1$ & & 273 & $34 \cdot 1$ & $28 \cdot 6$ & \\
\hline Male & 766 & $50 \cdot 5$ & $48 \cdot 1$ & 0.5 & 484 & 55.4 & $48 \cdot 6$ & 0.3 \\
\hline Qualifications & & & & 0.03 & & & & 0.2 \\
\hline $\begin{array}{l}\text { No qualifications (or in full-time } \\
\text { education) }\end{array}$ & 301 & $23 \cdot 1$ & $15 \cdot 1$ & & 138 & $23 \cdot 6$ & $15 \cdot 9$ & \\
\hline $\begin{array}{l}\text { School certificates \& other } \\
\text { qualifications }\end{array}$ & 856 & $50 \cdot 4$ & 51.4 & & 374 & $38 \cdot 1$ & $44 \cdot 8$ & \\
\hline Higher education below degree & 245 & $14 \cdot 0$ & 13.9 & & 97 & $6 \cdot 2$ & 11.5 & \\
\hline Degree & 321 & $12 \cdot 4$ & 19.5 & & 237 & $32 \cdot 1$ & $27 \cdot 8$ & \\
\hline In employment & 1280 & 74.7 & $77 \cdot 2$ & 0.5 & 609 & $70 \cdot 2$ & 73.7 & 0.5 \\
\hline Gross household income & & & & 0.03 & & & & 0.6 \\
\hline Lowest income group & 243 & $12 \cdot 7$ & $10 \cdot 6$ & & 123 & $8 \cdot 8$ & 13.4 & \\
\hline 2 & 267 & $15 \cdot 3$ & $12 \cdot 7$ & & 141 & 23.3 & $18 \cdot 4$ & \\
\hline 3 & 253 & $22 \cdot 2$ & $14 \cdot 2$ & & 85 & $14 \cdot 2$ & $10 \cdot 0$ & \\
\hline 4 & 381 & $20 \cdot 4$ & $24 \cdot 7$ & & 141 & 19.7 & $20 \cdot 2$ & \\
\hline Highest income group & 548 & 29.5 & 37.8 & & 239 & 33.9 & 38.0 & \\
\hline $\begin{array}{l}\text { Equalised household income } \\
(£) \text {, mean }\end{array}$ & NA & NA & NA & & 729 & 33040 & 32989 & 1.0 \\
\hline SD & & & & & & 23396 & 24057 & \\
\hline Equivalised household income & & & & & & & & 0.8 \\
\hline Lowest income quintile & NA & NA & NA & & 129 & $13 \cdot 8$ & $17 \cdot 6$ & \\
\hline 2 & & & & & 114 & $21 \cdot 4$ & $15 \cdot 7$ & \\
\hline 3 & & & & & 124 & $17 \cdot 8$ & $19 \cdot 1$ & \\
\hline 4 & & & & & 170 & 21.5 & $22 \cdot 3$ & \\
\hline Highest income quintile & & & & & 192 & 25.5 & $25 \cdot 3$ & \\
\hline Quintile of IMD score, England only & & & & & & & & 0.03 \\
\hline Most deprived & NA & NA & NA & & 131 & $27 \cdot 4$ & $14 \cdot 4$ & \\
\hline 2 & & & & & 142 & 8.7 & $19 \cdot 2$ & \\
\hline 3 & & & & & 157 & $15 \cdot 5$ & $18 \cdot 8$ & \\
\hline 4 & & & & & 138 & $18 \cdot 3$ & $16 \cdot 7$ & \\
\hline Least deprived & & & & & 133 & $17 \cdot 2$ & $14 \cdot 6$ & \\
\hline Other UK countries, not & & & & & 147 & $13 \cdot 0$ & $16 \cdot 3$ & \\
\hline Socio-economic classification $\ddagger$ & & & & 0.2 & & & & 0.6 \\
\hline Highest & 100 & $6 \cdot 6$ & $6 \cdot 0$ & & 361 & 43.7 & 44.5 & \\
\hline & 528 & $21 \cdot 7$ & 31.2 & & 170 & $16 \cdot 6$ & $20 \cdot 8$ & \\
\hline & 401 & $22 \cdot 9$ & 24.4 & & 300 & $39 \cdot 8$ & 34.7 & \\
\hline & 282 & 19.5 & 17.4 & & & & & \\
\hline & 283 & 19.5 & $15 \cdot 6$ & & & & & \\
\hline Lowest & 98 & $9 \cdot 8$ & $5 \cdot 3$ & & & & & \\
\hline Region & & & & 0.006 & & & & 0.2 \\
\hline Northern England & 451 & $23 \cdot 3$ & $27 \cdot 3$ & & 201 & 23.8 & 23.8 & \\
\hline Midlands & 266 & 13.4 & $15 \cdot 7$ & & 148 & $24 \cdot 2$ & 14.5 & \\
\hline London, East \& South England & 808 & $56 \cdot 6$ & 44.4 & & 352 & $39 \cdot 0$ & 45.5 & \\
\hline Scotland, Wales (+ NI in NDNS RP) & 199 & 6.7 & $12 \cdot 7$ & & 147 & $13 \cdot 0$ & $16 \cdot 3$ & \\
\hline $\begin{array}{l}\text { Number of adults in household, } \\
\text { mean }\end{array}$ & & $2 \cdot 15$ & $2 \cdot 27$ & 0.2 & & $2 \cdot 13$ & $2 \cdot 32$ & 0.07 \\
\hline SD & & 0.90 & 0.92 & & & 0.77 & 0.95 & \\
\hline Child in household, yes & 644 & $37 \cdot 3$ & 36.9 & 0.9 & 328 & 39.6 & $40 \cdot 1$ & 0.9 \\
\hline Marital status & & & & 0.06 & & & & 0.4 \\
\hline Single/never married & 358 & $17 \cdot 7$ & $20 \cdot 6$ & & 188 & $24 \cdot 8$ & $20 \cdot 0$ & \\
\hline $\begin{array}{l}\text { Married \& living together/ } \\
\text { cohabiting }\end{array}$ & 1075 & $67 \cdot 3$ & $70 \cdot 2$ & & 530 & $64 \cdot 2$ & $71 \cdot 2$ & \\
\hline Separated/divorced/widowed & 291 & $15 \cdot 0$ & $9 \cdot 2$ & & 130 & $11 \cdot 0$ & $8 \cdot 8$ & \\
\hline
\end{tabular}




\begin{tabular}{|c|c|c|c|c|c|c|c|c|}
\hline \multirow{3}{*}{$\begin{array}{l}\text { Adults: top } 10 \% \text { TFA } \\
\text { consumers }\end{array}$} & \multicolumn{3}{|c|}{ NDNS 2000/01 } & \multicolumn{5}{|c|}{ NDNS RP 2010/11 and 2011/12 } \\
\hline & $\begin{array}{c}\text { Total } \\
(N \text { 1724, } \\
\text { weighted } \\
N 1724)\end{array}$ & $\begin{array}{c}\text { Top 10\% } \\
\text { TFA } \\
\text { consumers } \\
\text { as \%FE } \\
(N 191, \\
\text { weighted } \\
N 176)\end{array}$ & $\begin{array}{c}\text { Remaining } \\
90 \% \\
(N 1533, \\
\text { weighted } \\
N 1548)\end{array}$ & & $\begin{array}{c}\text { Total } \\
(N 848, \\
\text { weighted } \\
N 1277)\end{array}$ & $\begin{array}{c}\text { Top 10\% } \\
\text { TFA } \\
\text { consumers } \\
\text { as \%FE } \\
(N 88, \\
\text { weighted } \\
N 130)\end{array}$ & $\begin{array}{c}\text { Remaining } \\
90 \% \\
(N 760 \\
\text { weighted } \\
N 1147)\end{array}$ & \\
\hline & Unweighted $n$ & $\% *$ & $\% *$ & $P$ value & Unweighted $n$ & $\% *$ & $\% *$ & $P$ value \\
\hline Ethnic group, two categories & & & & 0.5 & & & & 0.4 \\
\hline White & 1629 & $95 \cdot 6$ & 93.8 & & 750 & $81 \cdot 5$ & $85 \cdot 6$ & \\
\hline Non-White & 95 & 4.4 & $6 \cdot 2$ & & 98 & $18 \cdot 5$ & 14.4 & \\
\hline Ethnic group & & & & 0.6 & & & & 0.7 \\
\hline White & 1629 & $95 \cdot 6$ & $93 \cdot 8$ & & 750 & 81.5 & $85 \cdot 6$ & \\
\hline Black or Black British & 29 & 0.5 & $1 \cdot 7$ & & 29 & $6 \cdot 6$ & 3.7 & \\
\hline Asian or Asian British & 35 & $2 \cdot 6$ & $2 \cdot 6$ & & 40 & $6 \cdot 9$ & $6 \cdot 4$ & \\
\hline Any Other group incl. mixed & 31 & $1 \cdot 3$ & $2 \cdot 0$ & & 29 & $5 \cdot 0$ & $4 \cdot 4$ & \\
\hline Has longstanding illness, yes & 655 & $46 \cdot 7$ & 35.5 & 0.01 & 292 & 38.0 & 30.5 & 0.2 \\
\hline
\end{tabular}

$\% \mathrm{FE}$, percentage of food energy; NI, Northern Ireland; NA, not available.

*Values presented are percentages, unless indicated otherwise.

†Gross annual household income in the previous 12 months groupings for NDNS 2000/01 are: $<£ 8000, £ 8000$ to $<£ 12000$, £12000 to <£18000, £18000 to

$<£ 25000, \geq £ 25000$; for NDNS RP are: <£15000, £15000 to <£20000, £20000 to <£30000, £30000 to <£40000, $\geq £ 40000$.

¥Social class groups for NDNS 2000/01 are: I, professional; II, managerial and technical; IIIN, skilled non-manual; IIIM, skilled manual; IV, semi-skilled; V, unskilled; socio-economic classification grouping for NDNS RP are: Managerial \& Professional, Intermediate \& Small businesses, Routine \& Never worked.

\section{Analysis using predicted 'usual' intake distribution of trans-fatty acids}

The main results above were based on mean TFA intake over the seven or four assessment days. In separate analyses using the estimated usual intake distribution, we predicted that $36.6 \%$ in the pre-reformulation survey and none in the post-reformulation survey had TFA intake over the WHO recommendation. Having TFA intake above the WHO recommendation in the pre-reformulation survey, using this method, was associated with socio-economic disadvantage in having no qualifications or not having a degree, gross income and social class; but no significant differences were found for ethnicity (data not shown).

\section{Discussion}

Comparison of the NDNS results representing before (2000/01) and after product reformulation (2010-2012) shows that TFA consumption in the UK has reduced substantially between these periods. In Years 3 and 4 (2010-2012) of the recent NDNS RP, which incorporates product reformulation data, only $2.5 \%$ of adults consumed more than the current WHO recommended TFA limit (i.e. $\geq 1 \% \mathrm{TE}$ from TFA). This compares with $57 \%$ of adults in the earlier 2000/01 NDNS. The recent survey data indicate that almost all adults are now below the UK Dietary Reference Value of $2 \% \mathrm{FE}$. In addition, individuals who now have high TFA consumption tend to be consumers of products with a high natural TFA content such as butter and lamb. The range of TFA isomers derived from ruminant and industrial sources differs ${ }^{(32)}$. However, in studies based on replacing TFA with unsaturated fatty acids, improved lipid and lipoprotein profiles were observed for both iTFA and ruminant TFA replacement, potentially reducing CVD risk ${ }^{(4)}$. Prior to reformulation, high consumers generally consumed products containing iTFA. Although it appears that product reformulation has resulted in ruminant TFA becoming the dominant source of dietary TFA, the amount of ruminant products consumed on a population basis is less a cause for concern than for those containing iTFA, particularly as various positive nutrients are also gained from consuming ruminant products $^{(4,33)}$. Additionally, there appeared to be no major changes in percentage energy from food groups over the study period that would have explained the main differences in TFA intakes. Our results are in line with the NDNS 2008-2012 report which showed no evidence that individuals from households in lower quintiles of equivalised income had higher intakes than those in the top income quintile $^{(18)}$. Based on these results, which use average TFA values from composite product samples, it appears that voluntary product reformulation to reduce TFA consumption has been successful, with potential implications for CVD reduction.

Unadjusted analyses of the earlier 2000/01 NDNS showed that prior to reformulation, high TFA consumption was associated with lower income, lower education and having a long-term disability or illness; furthermore, having no qualifications compared with having a degree remained an independent predictor of being a top $10 \%$ TFA consumer in the multivariate analyses. Given that higher TFA consumption is linked to higher CHD risk ${ }^{(2)}$, our results indicate that prior to reformulation the products' TFA content and consumption patterns within society were likely to lead to health inequalities. 
Table 3 Odds of being in the top $10 \%$ of trans-fatty acid (TFA) consumers as a percentage of food energy compared with the remaining $90 \%$ depending on sociodemographic characteristics of adults (aged 19-64 years) in the National Diet and Nutrition Survey (NDNS 2000/ 01) and Years 3 and 4 of the National Diet and Nutrition Survey Rolling Programme (NDNS RP 2010/11 and 2011/12)

\begin{tabular}{|c|c|c|c|c|c|c|}
\hline \multirow[b]{3}{*}{ Adult: multivariate analyses top $10 \%$ TFA consumers } & \multicolumn{6}{|c|}{ Adjusted OR and $95 \% \mathrm{Cl}$ of being in the top $10 \%$ TFA consumers as $\% \mathrm{FE}$} \\
\hline & \multicolumn{3}{|c|}{$\begin{array}{c}\text { NDNS 2000/01 } \\
(N \text { 1689, weighted } N \text { 1680) }\end{array}$} & \multicolumn{3}{|c|}{$\begin{array}{l}\text { NDNS RP } 2010 / 11 \text { and } 2011 / 12 \\
(N 728, \text { weighted } N 1068)\end{array}$} \\
\hline & OR & $95 \% \mathrm{Cl}$ & $P$ value & OR & $95 \% \mathrm{Cl}$ & $P$ value \\
\hline Food energy intake per 418 kJ (100 kcal) & 1.01 & $1.01,1.01$ & $<0.001$ & 1.00 & $0.95,1.06$ & 0.9 \\
\hline Age (per year) & 1.00 & $0.99,1.02$ & 0.9 & 1.02 & $0.98,1.05$ & 0.3 \\
\hline \multicolumn{7}{|l|}{ Gender } \\
\hline Male & 1.00 & Ref. & - & 1.00 & Ref. & - \\
\hline Female & 0.76 & $0.50,1.16$ & 0.2 & 0.85 & $0.47,1.55$ & 0.6 \\
\hline \multicolumn{7}{|l|}{ Qualification } \\
\hline No qualifications (or in full-time education) & 1.00 & Ref. & $0.1 \dagger$ & 1.00 & Ref. & $0.2 \dagger$ \\
\hline School certificates \& other qualifications & 0.74 & $0.48,1.14$ & 0.3 & 0.43 & $0.18,1.04$ & 0.1 \\
\hline Higher education below degree & 0.74 & $0.40,1.35$ & 0.2 & 0.27 & $0.07,1.03$ & 0.3 \\
\hline Degree & 0.51 & $0.28,0.92$ & 0.03 & 0.61 & $0.23,1.60$ & 0.3 \\
\hline \multicolumn{7}{|l|}{ Gross household income* } \\
\hline Lowest income group & 1.00 & Ref. & $0.06 \dagger$ & 1.00 & Ref. & $0.05 \dagger$ \\
\hline 2 & 0.88 & $0.47,1.64$ & 0.7 & 2.78 & $1 \cdot 23,6 \cdot 22$ & 0.01 \\
\hline 3 & 1.16 & 0.652 .09 & 0.6 & 3.25 & $1 \cdot 10,9.62$ & 0.03 \\
\hline 4 & 0.57 & $0.31,1.05$ & 0.07 & 2.49 & $1.08,5 \cdot 75$ & 0.03 \\
\hline Highest income group & 0.60 & $0 \cdot 31,1 \cdot 17$ & $0 \cdot 1$ & $2 \cdot 57$ & $1 \cdot 08,6 \cdot 14$ & 0.03 \\
\hline \multicolumn{7}{|l|}{ Region } \\
\hline London, East \& South England & 1.00 & Ref. & - & 1.00 & Ref. & - \\
\hline North England & 0.53 & $0.33,0.85$ & 0.008 & 1.39 & $0.66,2.94$ & 0.4 \\
\hline Midlands & 0.65 & $0.39,1.07$ & 0.09 & $2 \cdot 40$ & $1 \cdot 13,5 \cdot 11$ & 0.02 \\
\hline Scotland, Wales (+NI in NDNS RP) & 0.33 & $0.19,0.57$ & $<0.001$ & $1 \cdot 19$ & $0.48,2.92$ & 0.7 \\
\hline \multicolumn{7}{|l|}{ Ethnic group } \\
\hline White & 1.00 & Ref. & - & 1.00 & Ref. & - \\
\hline Black or Black British & 0.24 & $0.03,2 \cdot 15$ & 0.2 & 1.22 & $0.24,6 \cdot 23$ & 0.8 \\
\hline Asian or Asian British & 0.84 & $0.26,2.73$ & 0.8 & 1.54 & $0.44,5.42$ & 0.5 \\
\hline Other incl. mixed & 0.74 & $0.20,2 \cdot 77$ & 0.7 & 1.34 & $0.30,6.04$ & 0.7 \\
\hline \multicolumn{7}{|l|}{ Marital status } \\
\hline Single/never married & 1.00 & Ref. & - & 1.00 & Ref. & - \\
\hline Married \& living together/cohabiting & 1.00 & $0.57,1.75$ & 1.0 & 0.56 & $0.21,1.48$ & 0.2 \\
\hline Separated/divorced/widowed & 1.49 & $0.85,2.64$ & 0.2 & 0.71 & $0.25,2.02$ & 0.5 \\
\hline \multicolumn{7}{|l|}{ Long-term illness or disability } \\
\hline Yes & 1.00 & Ref. & - & 1.00 & Ref. & - \\
\hline No & 0.70 & $0.49,1.01$ & 0.06 & 0.71 & $0.41,1.23$ & 0.2 \\
\hline Number of adults in household & 0.96 & $0.78,1.23$ & 0.7 & 0.83 & $0.57,1.20$ & 0.3 \\
\hline Number of children in household & 1.06 & $0.89,1.26$ & 0.5 & 1.07 & $0.81,1.39$ & 0.6 \\
\hline
\end{tabular}

$\% \mathrm{FE}$, percentage of food energy; NI, Northern Ireland; Ref., reference category.

*Gross annual household income in the previous 12 months groupings for NDNS 2000/01 are: $<£ 8000$, £8000 to $<£ 12000, £ 12000$ to $<£ 18000$, £18000 to $<£ 25000, \geq £ 25000$; for NDNS RP are: $<£ 15000, £ 15000$ to $<£ 20000, £ 20000$ to $<£ 30000, £ 30000$ to $<£ 40000, \geq £ 40000$.

†Test for trend across the groups.

Post-reformulation results from the NDNS RP (2010-2012) were less clear regarding education and income-related inequalities. The sample size was smaller in the post-reformulation survey, with lower power to find associations, and about a third more held a degree, which may have diluted any association. However, the multivariate analyses indicated that higher income groups are now more likely to be top $10 \%$ TFA consumers than the lowest income group. High TFA consumers now tend to consume products with a high natural TFA content such as butter, lamb and cheese, which are relatively expensive products more affordable to higher income groups. Nevertheless, in the unadjusted analyses of IMD score in the post-reformulation NDNS, the top $10 \%$ consumers were more likely to be living in the most deprived areas of England than the remaining $90 \%$. In addition to income, IMD incorporates other domains, including barriers to housing, services and employment. One explanation for our findings is that remote areas in the UK are generally considered deprived $^{(34)}$ and residents may also have more traditional diets high in natural TFA.

\section{Strengths and limitations}

A strength of the present study is the use of UK nationally representative surveys with TFA data from both before and after industrial reformulation to reduce the TFA content of food products. There were also similarities in data-gathering methods and variables produced from the NDNS data sets. However, the results are limited by self-reporting of intake, particularly in the NDNS RP (2010-2012) where individuals did not weigh their food intake and documented fewer intake days ${ }^{(18)}$. Weighed diet records are more accurate regarding portion size estimation than food diaries. Both have a high respondent 
Table 4 Percentage contribution of main food groups to energy intake and average daily trans-fatty acid (TFA) intake of adults (aged 19-64 years) in the National Diet and Nutrition Survey (NDNS 2000/01) and Years 3 and 4 of the National Diet and Nutrition Survey Rolling Programme (NDNS RP 2010/11 and 2011/12) who consumed $1 \%$ or more of their total energy intake from TFA compared with those who consumed less

\begin{tabular}{|c|c|c|c|c|c|c|c|c|}
\hline \multirow{3}{*}{$\begin{array}{l}\text { Adults: WHO TFA limit } \\
\text { Main food group name }\end{array}$} & \multicolumn{4}{|c|}{ NDNS 2000/01 } & \multicolumn{4}{|c|}{ NDNS RP 2010/11 and 2011/12 } \\
\hline & \multirow{2}{*}{$\begin{array}{c}\text { Total } \\
(N 1724, \\
\text { weighted } \\
N 1724) \\
\% \text { FE }\end{array}$} & \multirow{2}{*}{$\begin{array}{c}\geq 1 \% \text { TE } \\
\text { from TFA } \\
(N 988, \\
\text { weighted } \\
N 988)\end{array}$} & \multirow{2}{*}{$\begin{array}{c}<1 \% \text { TE } \\
\text { from TFA } \\
(N 736, \\
\text { weighted } \\
N 736)\end{array}$} & \multirow{2}{*}{$\begin{array}{l}P \text { value for } \Delta \\
\text { in } \% \text { TFA }\end{array}$} & $\begin{array}{c}\text { Total } \\
(N 848, \\
\text { weighted } \\
N 1277)\end{array}$ & $\begin{array}{c}\geq 1 \% \text { TE } \\
\text { from TFA } \\
(N 22, \\
\text { weighted } \\
N 32)\end{array}$ & $\begin{array}{c}<1 \% \text { TE } \\
\text { from TFA } \\
(N 826, \\
\text { weighted } \\
N 1245)\end{array}$ & \multirow{2}{*}{$\begin{array}{l}P \text { value for } \Delta \\
\text { in } \% \text { TFA }\end{array}$} \\
\hline & & & & & $\% F E$ & $\%$ TFA & $\%$ TFA & \\
\hline $\begin{array}{l}\text { Pasta, rice and other } \\
\text { cereals }\end{array}$ & $6 \cdot 3$ & $2 \cdot 8$ & 3.5 & 0.06 & 8.9 & 0.3 & 3.4 & $<0.001$ \\
\hline White bread & 8.9 & 0.9 & 1.4 & 0.05 & $7 \cdot 7$ & 0.8 & $3 \cdot 0$ & $<0.001$ \\
\hline Wholemeal bread & $2 \cdot 0$ & 0.2 & 0.5 & $<0.001$ & $2 \cdot 6$ & 0.4 & $1 \cdot 1$ & 0.001 \\
\hline $\begin{array}{l}\text { Brown, granary and } \\
\text { wheatgerm }\end{array}$ & & & & & 2.5 & 0.3 & 0.6 & 0.004 \\
\hline Other bread & $2 \cdot 7$ & 0.8 & 1.2 & $<0.001$ & 0.2 & 0.0 & $0 \cdot 1$ & $<0.001$ \\
\hline High-fibre breakfast cereals & 3.0 & 1.1 & 0.8 & 0.2 & $2 \cdot \overline{2}$ & 0.0 & 0.2 & $<0.001$ \\
\hline Biscuits & $2 \cdot 8$ & $8 \cdot 6$ & 5.5 & $<0.001$ & $3 \cdot 4$ & 0.3 & $1 \cdot 1$ & $<0.001$ \\
\hline $\begin{array}{l}\text { Buns, cakes, pastries \& } \\
\text { fruit pies }\end{array}$ & $3 \cdot 8$ & $9 \cdot 9$ & $6 \cdot 7$ & $<0.001$ & $3 \cdot 3$ & $6 \cdot 1$ & $4 \cdot 1$ & 0.5 \\
\hline Puddings & $1 \cdot 1$ & $2 \cdot 0$ & 1.2 & $<0.001$ & 0.8 & $6 \cdot 0$ & 1.5 & 0.2 \\
\hline Whole milk & 1.7 & $1 \cdot 3$ & 1.8 & 0.03 & 0.9 & 1.0 & $1 \cdot 7$ & 0.2 \\
\hline Semi-skimmed milk & $3 \cdot 1$ & $3 \cdot 2$ & 5.5 & $<0.001$ & $2 \cdot 6$ & 3.5 & 7.8 & $<0.001$ \\
\hline Other milk and cream & 0.6 & 1.0 & 0.9 & 0.2 & 0.9 & $10 \cdot 3$ & 2.4 & 0.05 \\
\hline Cheese & 3.0 & $7 \cdot 2$ & 9.6 & $<0.001$ & 3.4 & $17 \cdot 8$ & $17 \cdot 8$ & 1.0 \\
\hline $\begin{array}{l}\text { Yoghurt, fromage frais and } \\
\text { dairy }\end{array}$ & $1 \cdot 2$ & $0 \cdot 6$ & $1 \cdot 1$ & $<0.001$ & 1.4 & $1 \cdot 3$ & 1.9 & 0.3 \\
\hline Eggs and egg dishes & $2 \cdot 2$ & $2 \cdot 4$ & $3 \cdot 3$ & 0.002 & $2 \cdot 0$ & 0.8 & $2 \cdot 1$ & $<0.001$ \\
\hline Butter & 1.3 & 4.0 & 3.9 & 0.9 & 1.4 & $11 \cdot 1$ & $7 \cdot 1$ & 0.2 \\
\hline PUFA margarine \& oils & 0.1 & 0.5 & 0.4 & 0.9 & 0.2 & 0.0 & 0.0 & \\
\hline Low-fat spread & 0.4 & 1.6 & 1.9 & 0.5 & 0.3 & 0.0 & 0.2 & $<0.001$ \\
\hline $\begin{array}{l}\text { Other margarine fats and } \\
\text { oils }\end{array}$ & 0.6 & 4.5 & $3 \cdot 3$ & 0.02 & 0.8 & 0.1 & $0 \cdot 1$ & 0.9 \\
\hline Reduced-fat spread & 1.8 & 8.7 & $4 \cdot 1$ & $<0.001$ & $2 \cdot 0$ & 0.2 & 1.5 & $<0.001$ \\
\hline Bacon and ham & 1.6 & 0.3 & 0.6 & $<0.001$ & 1.6 & 0.2 & 1.0 & $<0.001$ \\
\hline Beef, veal and dishes & $2 \cdot 9$ & 3.7 & $6 \cdot 3$ & $<0.001$ & 2.5 & 3.2 & $8 \cdot 1$ & $<0.001$ \\
\hline Lamb and dishes & 0.8 & 2.5 & $2 \cdot 9$ & 0.4 & 0.8 & $14 \cdot 3$ & $4 \cdot 2$ & 0.05 \\
\hline Pork and dishes & 0.9 & 0.2 & 0.4 & 0.001 & 1.0 & 0.4 & 0.6 & 0.3 \\
\hline Coated chicken & 1.0 & $1 \cdot 1$ & 1.4 & 0.1 & 0.9 & 0.0 & 0.7 & $<0.001$ \\
\hline Chicken and turkey dishes & 3.9 & 1.2 & 3.0 & $<0.001$ & $4 \cdot 3$ & $2 \cdot 1$ & 4.0 & 0.03 \\
\hline Burgers and kebabs & 1.2 & $2 \cdot 3$ & $2 \cdot 6$ & 0.4 & 1.0 & 8.5 & $2 \cdot 7$ & 0.4 \\
\hline Sausages & 1.4 & 0.5 & 0.9 & $<0.001$ & 1.7 & 0.8 & $2 \cdot 1$ & $<0.001$ \\
\hline Meat pies and pastries & $2 \cdot 3$ & $7 \cdot 6$ & 3.9 & $<0.001$ & 1.8 & 0.5 & $1 \cdot 7$ & 0.001 \\
\hline $\begin{array}{l}\text { Other meat and meat } \\
\text { products }\end{array}$ & 0.7 & 0.7 & $1 \cdot 1$ & 0.02 & 0.5 & $1 \cdot 8$ & 1.0 & 0.6 \\
\hline White fish coated or fried & 1.2 & $2 \cdot 0$ & $2 \cdot 1$ & 0.6 & $1 \cdot 2$ & 0.9 & 1.8 & 0.2 \\
\hline Other white fish, shellfish & 0.6 & 0.4 & 0.6 & 0.04 & 0.9 & 0.1 & 0.3 & 0.06 \\
\hline Oily fish & 1.2 & 0.3 & 0.5 & 0.06 & 1.0 & 0.0 & 0.2 & 0.01 \\
\hline Vegetables not raw & 3.8 & 1.3 & 1.7 & 0.09 & 4.0 & 0.9 & 1.5 & 0.3 \\
\hline $\begin{array}{l}\text { Chips fried \& roast } \\
\text { potatoes }\end{array}$ & $5 \cdot 3$ & 4.4 & $4 \cdot 2$ & 0.7 & 4.4 & $1 \cdot 2$ & $2 \cdot 7$ & 0.08 \\
\hline $\begin{array}{l}\text { Other potatoes, potato } \\
\text { salads }\end{array}$ & 3.0 & 0.9 & 1.0 & 0.3 & $2 \cdot 7$ & 0.2 & 0.4 & 0.1 \\
\hline Crisps and savoury snacks & $2 \cdot 0$ & $1 \cdot 3$ & 1.3 & 0.9 & $2 \cdot 1$ & 0.0 & 0.5 & $<0.001$ \\
\hline Chocolate confectionery & 2.4 & 3.5 & $2 \cdot 8$ & 0.06 & $2 \cdot 1$ & 0.3 & $2 \cdot 2$ & $<0.001$ \\
\hline Miscellaneous & 3.0 & $2 \cdot 6$ & 3.5 & $<0.001$ & 3.9 & 3.0 & $3 \cdot 8$ & 0.7 \\
\hline Ice cream & 0.6 & 1.3 & 1.4 & 0.7 & 0.6 & 0.6 & 1.4 & 0.05 \\
\hline
\end{tabular}

\%TE, percentage of total energy; \%FE, percentage of food energy; \%TFA, percentage of TFA intake; TFA intake $\geq 1 \%$ TE is above the WHO recommended TFA limit.

Percentage contributions of TFA from food types are weighted means of the individuals' percentage contributions.

Food groups contributing less than $0.5 \%$ TFA to food energy in all TFA groups were not tabled.

burden so can induce significant changes in individual behaviour, but weighed diet records are more invasive and more likely to influence individual choices. For instance, they may encourage more consumption of prepared foods easy to weigh and less eating out ${ }^{(35)}$. There has been evidence of under-reporting in the NDNS 2000/01 in relation to energy needs ${ }^{(36)}$, where respondents were asked to weigh their intake over $7 \mathrm{~d}$. In addition, doubly labelled water feasibility studies conducted for both the earlier UK NDNS and the NDNS RP point towards under-reporting ${ }^{(37,38)}$. It is likely that under-reporting and inaccuracies are greater in people with lower socio- 
Table 5 Percentage contribution of main food groups to energy intake and average daily trans-fatty acid (TFA) intake of adults (aged 19-64 years) in the National Diet and Nutrition Survey (NDNS 2000/01) and Years 3 and 4 of the National Diet and Nutrition Survey Rolling Programme (NDNS RP 2010/11 and 2011/12) who were the top 10\% of TFA consumers as a percentage of food energy compared with the remaining $90 \%$

\begin{tabular}{|c|c|c|c|c|c|c|c|c|}
\hline \multirow{3}{*}{$\begin{array}{l}\text { Adults: top } 10 \% \text { TFA } \\
\text { consumers } \\
\text { Main food group } \\
\text { name }\end{array}$} & \multicolumn{4}{|c|}{ NDNS 2000/01 } & \multicolumn{4}{|c|}{ NDNS RP $2010 / 11$ and $2011 / 12$} \\
\hline & $\begin{array}{c}\text { Total } \\
(N 1724, \\
\text { weighted } \\
N 1724)\end{array}$ & $\begin{array}{c}\text { Top 10\% } \\
\text { TFA } \\
\text { consumers } \\
\text { as } \% F E \\
(N 191, \\
\text { weighted } \\
N 176)\end{array}$ & $\begin{array}{c}\text { Remaining } \\
90 \% \\
(N 1533 \\
\text { weighted } \\
N 1548)\end{array}$ & \multirow{2}{*}{$\begin{array}{l}P \text { value for } \Delta \\
\text { in \%TFA }\end{array}$} & $\begin{array}{c}\text { Total } \\
(N \text { 848, } \\
\text { weighted } \\
N 1277)\end{array}$ & $\begin{array}{c}\text { Top 10\% } \\
\text { TFA } \\
\text { consumers } \\
\text { as \%FE } \\
(N 88, \\
\text { weighted } \\
N 130)\end{array}$ & $\begin{array}{c}\text { Remaining } \\
90 \% \\
(N 760 \\
\text { weighted } \\
N 1147)\end{array}$ & \multirow{2}{*}{$\begin{array}{l}P \text { value for } \Delta \\
\text { in \%TFA }\end{array}$} \\
\hline & $\% F E$ & $\%$ TFA & $\%$ TFA & & $\% F E$ & $\%$ TFA & $\%$ TFA & \\
\hline $\begin{array}{l}\text { Pasta, rice and other } \\
\text { cereals }\end{array}$ & $6 \cdot 3$ & 1.8 & $3 \cdot 3$ & $<0.001$ & $8 \cdot 9$ & $3 \cdot 1$ & $3 \cdot 4$ & $0 \cdot 7$ \\
\hline White bread & 8.9 & 0.6 & 1.2 & $<0.001$ & $7 \cdot 7$ & 0.9 & $3 \cdot 2$ & $<0.001$ \\
\hline Wholemeal bread & $2 \cdot 0$ & 0.1 & 0.4 & $<0.001$ & $2 \cdot 6$ & 1.5 & 1.0 & 0.7 \\
\hline $\begin{array}{l}\text { Brown, granary and } \\
\text { wheatgerm }\end{array}$ & & & & & 2.5 & 0.3 & 0.6 & 0.003 \\
\hline Other bread & $2 \cdot 7$ & 0.4 & 1.0 & $<0.001$ & 0.2 & 0.0 & $0 \cdot 1$ & 0.05 \\
\hline $\begin{array}{l}\text { High-fibre breakfast } \\
\text { cereals }\end{array}$ & $3 \cdot 0$ & 0.9 & $1 \cdot 0$ & 0.9 & $2 \cdot 2$ & 0.2 & 0.2 & 0.5 \\
\hline Biscuits & $2 \cdot 8$ & $12 \cdot 4$ & 6.7 & $<0.001$ & 3.4 & 0.8 & $1 \cdot 1$ & 0.2 \\
\hline $\begin{array}{l}\text { Buns, cakes, pastries \& } \\
\text { fruit pies }\end{array}$ & 3.8 & $10 \cdot 2$ & $8 \cdot 3$ & $0 \cdot 1$ & $3 \cdot 3$ & $5 \cdot 3$ & $4 \cdot 0$ & 0.4 \\
\hline Puddings & 1.1 & 1.5 & $1 \cdot 7$ & 0.5 & 0.8 & $3 \cdot 1$ & 1.4 & $0 \cdot 1$ \\
\hline Whole milk & 1.7 & 0.9 & 1.6 & $<0.001$ & 0.9 & 1.5 & 1.7 & 0.6 \\
\hline Semi-skimmed milk & 3.1 & $2 \cdot 2$ & 4.4 & $<0.001$ & $2 \cdot 6$ & $3 \cdot 1$ & $8 \cdot 1$ & $<0.001$ \\
\hline Other milk and cream & 0.6 & 0.9 & 1.0 & 0.7 & 0.9 & $6 \cdot 1$ & $2 \cdot 2$ & 0.01 \\
\hline Cheese & 3.0 & 5.4 & 8.6 & $<0.001$ & 3.4 & $19 \cdot 2$ & $17 \cdot 6$ & 0.4 \\
\hline $\begin{array}{l}\text { Yoghurt, fromage frais } \\
\text { and dairy }\end{array}$ & 1.2 & 0.3 & $0 \cdot 8$ & $<0.001$ & 1.4 & 0.9 & $2 \cdot 0$ & 0.001 \\
\hline Eggs and egg dishes & $2 \cdot 2$ & 1.8 & $2 \cdot 9$ & $<0.001$ & $2 \cdot 0$ & 1.5 & $2 \cdot 1$ & 0.09 \\
\hline Butter & 1.3 & $2 \cdot 1$ & $4 \cdot 1$ & $<0.001$ & 1.4 & $13 \cdot 9$ & $6 \cdot 4$ & $<0.001$ \\
\hline PUFA margarine \& oils & 0.1 & $0 \cdot 1$ & 0.5 & 0.005 & 0.2 & 0.0 & 0.0 & \\
\hline Low-fat spread & 0.4 & 1.5 & 1.8 & 0.6 & 0.3 & 0.0 & 0.2 & $<0.001$ \\
\hline $\begin{array}{l}\text { Other margarine fats } \\
\text { and oils }\end{array}$ & 0.6 & $6 \cdot 3$ & $3 \cdot 7$ & 0.3 & 0.8 & 0.0 & 0.2 & 0.2 \\
\hline Reduced-fat spread & 1.8 & 14.8 & 5.8 & $<0.001$ & $2 \cdot 0$ & 0.5 & 1.6 & $<0.001$ \\
\hline Bacon and ham & 1.6 & 0.2 & 0.4 & $<0.001$ & 1.6 & 0.5 & 1.0 & $<0.001$ \\
\hline Beef, veal and dishes & $2 \cdot 9$ & 2.5 & $5 \cdot 1$ & $<0.001$ & 2.5 & 5.4 & $8 \cdot 3$ & 0.008 \\
\hline Lamb and dishes & 0.8 & $2 \cdot 0$ & $2 \cdot 8$ & 0.9 & 0.8 & 8.4 & 3.9 & 0.01 \\
\hline Pork and dishes & 0.9 & 0.1 & 0.3 & $<0.001$ & 1.0 & 0.3 & 0.7 & 0.003 \\
\hline Coated chicken & 1.0 & 0.8 & 1.3 & 0.003 & 0.9 & 0.2 & 0.7 & $<0.001$ \\
\hline $\begin{array}{l}\text { Chicken and turkey } \\
\text { dishes }\end{array}$ & 3.9 & 0.6 & $2 \cdot 1$ & $<0.001$ & $4 \cdot 3$ & 1.4 & $4 \cdot 2$ & $<0.001$ \\
\hline Burgers and kebabs & 1.2 & $2 \cdot 0$ & 2.5 & 0.3 & 1.0 & $4 \cdot 2$ & $2 \cdot 7$ & 0.5 \\
\hline Sausages & 1.4 & 0.3 & 0.7 & $<0.001$ & 1.7 & 1.0 & $2 \cdot 2$ & $<0.001$ \\
\hline Meat pies and pastries & $2 \cdot 3$ & $10 \cdot 7$ & 5.5 & $<0.001$ & 1.8 & 0.8 & $1 . \overline{7}$ & 0.03 \\
\hline $\begin{array}{l}\text { Other meat and meat } \\
\text { products }\end{array}$ & 0.7 & 0.6 & 0.9 & 0.02 & 0.5 & $1 \cdot 3$ & $1 \cdot 0$ & 0.7 \\
\hline $\begin{array}{l}\text { White fish coated or } \\
\text { fried }\end{array}$ & $1 \cdot 2$ & $2 \cdot 0$ & $2 \cdot 1$ & 0.9 & $1 \cdot 2$ & 1.5 & $1 \cdot 8$ & 0.6 \\
\hline $\begin{array}{l}\text { Other white fish, } \\
\text { shellfish }\end{array}$ & 0.6 & 0.2 & 0.5 & 0.001 & 0.9 & 0.1 & 0.3 & 0.008 \\
\hline Oily fish & 1.2 & 0.3 & 0.4 & 0.6 & 1.0 & 0.8 & $0 \cdot 1$ & 0.4 \\
\hline Vegetables not raw & $3 \cdot 8$ & 1.0 & 1.5 & 0.2 & $4 \cdot 0$ & $2 \cdot 3$ & 1.4 & 0.3 \\
\hline $\begin{array}{l}\text { Chips fried \& roast } \\
\text { potatoes }\end{array}$ & $5 \cdot 3$ & 3.8 & $4 \cdot 4$ & $0 . \overline{2}$ & 4.4 & $2 \cdot 7$ & $2 \cdot 7$ & $1 \cdot 0$ \\
\hline $\begin{array}{l}\text { Other potatoes, potato } \\
\text { salads }\end{array}$ & $3 \cdot 0$ & $1 \cdot 0$ & 0.9 & 0.9 & $2 \cdot 7$ & 0.3 & 0.4 & 0.2 \\
\hline $\begin{array}{l}\text { Crisps and savoury } \\
\text { snacks }\end{array}$ & $2 \cdot 0$ & $1 \cdot 1$ & $1 \cdot 3$ & 0.5 & $2 \cdot 1$ & 0.2 & 0.5 & $<0.001$ \\
\hline $\begin{array}{l}\text { Chocolate } \\
\text { confectionery }\end{array}$ & $2 \cdot 4$ & $3 \cdot 1$ & $3 \cdot 2$ & $0 \cdot 8$ & $2 \cdot 1$ & $1 \cdot 7$ & $2 \cdot 2$ & 0.4 \\
\hline Miscellaneous & 3.0 & 1.6 & $3 \cdot 1$ & $<0.001$ & 3.9 & $3 \cdot 2$ & 3.8 & 0.4 \\
\hline Ice cream & 0.6 & 0.8 & 1.4 & $<0.001$ & 0.6 & 0.8 & 1.5 & 0.07 \\
\hline
\end{tabular}

\%FE, percentage of food energy; \%TFA, percentage of TFA intake.

Percentage contributions of TFA from food types are weighted means of the individuals' percentage contributions.

Food groups contributing less than $0.5 \%$ TFA to food energy in all TFA groups were not tabled. 
Table 6 Nutrient intakes of adults (aged 19-64 years) in the National Diet and Nutrition Survey (NDNS 2000/01) and Years 3 and 4 of the National Diet and Nutrition Survey Rolling Programme (NDNS RP 2010/11 and 2011/12) who consumed $1 \%$ or more of their total energy intake from trans-fatty acids (TFA) compared with those who consumed less

\begin{tabular}{|c|c|c|c|c|c|c|c|c|c|c|}
\hline \multirow[b]{3}{*}{ Adults: WHO TFA limit } & \multicolumn{5}{|c|}{ NDNS 2000/01 } & \multicolumn{5}{|c|}{ NDNS RP 2010/11 and 2011/12 } \\
\hline & \multicolumn{2}{|c|}{$\begin{array}{c}\geq 1 \% \text { TE from TFA } \\
(N \text { 988, weighted } N \text { 988) }\end{array}$} & \multicolumn{2}{|c|}{$\begin{array}{c}<1 \% \text { TE from TFA } \\
(N 736, \text { weighted } N 736)\end{array}$} & \multirow[b]{2}{*}{$P$ value } & \multicolumn{2}{|c|}{$\begin{array}{c}\geq 1 \% \text { TE from TFA } \\
(N 22, \text { weighted } N 32)\end{array}$} & \multicolumn{2}{|c|}{$\begin{array}{c}<1 \% \text { TE from TFA } \\
(N 826, \text { weighted } N 1245)\end{array}$} & \multirow[b]{2}{*}{$P$ value } \\
\hline & Mean & SD & Mean & SD & & Mean & SD & Mean & SD & \\
\hline TFA (g) & $3 \cdot 11$ & 1.30 & 1.59 & 0.66 & $<0.001$ & $2 \cdot 18$ & 0.62 & 1.02 & 0.53 & $<0.001$ \\
\hline TFA (\%TE) & 1.38 & 0.34 & 0.75 & 0.18 & $<0.001$ & $1 \cdot 20$ & 0.26 & 0.49 & 0.20 & $<0.001$ \\
\hline TFA (\%FE) & 1.43 & 0.34 & 0.81 & 0.20 & $<0.001$ & 1.25 & 0.26 & 0.52 & 0.20 & $<0.001$ \\
\hline Total energy (kJ/d) & 8452 & 2582 & 7866 & 2427 & $<0.001$ & 6891 & 1657 & 7673 & 2335 & 0.04 \\
\hline Total energy $(\mathrm{kcal} / \mathrm{d})$ & 2020 & 617 & 1880 & 580 & $<0.001$ & 1647 & 396 & 1834 & 558 & 0.04 \\
\hline Food energy $(\mathrm{kJ} / \mathrm{d})$ & 7991 & 2439 & 7071 & 2117 & $<0.001$ & 6643 & 1590 & 7284 & 2180 & 0.07 \\
\hline Food energy $(\mathrm{kcal} / \mathrm{d})$ & 1910 & 583 & 1690 & 506 & $<0.001$ & 1589 & 380 & 1741 & 521 & 0.07 \\
\hline Fat (\%FE) & 37.3 & 5.4 & $32 \cdot 7$ & 6.0 & $<0.001$ & 41.0 & 4.9 & 34.2 & 6.1 & $<0.001$ \\
\hline Saturated fat (\%FE) & 14.4 & 3.0 & 11.8 & 2.7 & $<0.001$ & 18.4 & 3.8 & $12 \cdot 3$ & $3 \cdot 1$ & $<0.001$ \\
\hline NMES (\%FE) & 12.5 & 6.2 & $13 \cdot 0$ & 7.5 & 0.2 & 9.4 & 5.3 & $12 \cdot 0$ & 6.4 & 0.05 \\
\hline $\mathrm{Na}(\mathrm{mg} / 4184 \mathrm{~kJ}(1000 \mathrm{kcal}))$ & 1520 & 324 & 1595 & 398 & $<0.001$ & 1150 & 435 & 1295 & 336 & 0.2 \\
\hline Vitamin C (mg/4184 kJ (1000 kcal)) & 41.0 & 26.7 & 55.2 & $35 \cdot 0$ & $<0.001$ & 42.4 & 22.6 & $46 \cdot 3$ & $36 \cdot 2$ & 0.5 \\
\hline Vitamin D $(\mu \mathrm{g} / 4184 \mathrm{~kJ}(1000 \mathrm{kcal}))$ & 1.7 & $1 \cdot 1$ & 2.0 & 1.5 & $<0.001$ & 1.3 & 0.7 & 1.6 & 1.1 & 0.02 \\
\hline Vitamin E (mg/4184 kJ (1000 kcal)) & 4.9 & 1.5 & 5.4 & 1.9 & $<0.001$ & 4.5 & 1.4 & 5.5 & 2.3 & $<0.001$ \\
\hline $\mathrm{F} \& \mathrm{~V}^{*}(\mathrm{~g} / 4184 \mathrm{~kJ}(1000 \mathrm{kcal}))$ & 99.7 & 7.4 & 142 & 108 & $<0.001$ & 141 & $89 \cdot 3$ & 168.8 & 112 & 0.2 \\
\hline
\end{tabular}

\%TE, percentage of total energy; \%FE, percentage of food energy; NMES, non-milk extrinsic sugars; F\&V, fruit and vegetables; TFA intake $\geq 1 \%$ TE is above the WHO recommended TFA limit.

*Total grams of $F \& V$ do not include pulses, baked beans and fruit juice.

Table 7 Nutrient intake of adults (aged 19-64 years) in the National Diet and Nutrition Survey (NDNS 2000/01) and Years 3 and 4 of the National Diet and Nutrition Survey Rolling Programme (NDNS PR 2010/11 and 2011/12) who were the top $10 \%$ of trans-fatty acid consumers as a percentage of food energy compared with the remaining $90 \%$

\begin{tabular}{|c|c|c|c|c|c|c|c|c|c|c|}
\hline \multirow[b]{3}{*}{ Adults: top $10 \%$ TFA consumers } & \multicolumn{5}{|c|}{ NDNS 2000/01 } & \multicolumn{5}{|c|}{ NDNS RP 2010/11 and 2011/12 } \\
\hline & \multicolumn{2}{|c|}{$\begin{array}{l}\text { Top } 10 \% \text { TFA consumers as } \% F E \\
(N 191 \text {, weighted } N 176)\end{array}$} & \multicolumn{2}{|c|}{$\begin{array}{c}\text { Remaining } 90 \% \\
(N 1533, \text { weighted } N 1548)\end{array}$} & \multirow[b]{2}{*}{$P$ value } & \multicolumn{2}{|c|}{$\begin{array}{c}\text { Top } 10 \% \text { TFA consumers as \%FE } \\
(N 88, \text { weighted } N 130)\end{array}$} & \multicolumn{2}{|c|}{$\begin{array}{c}\text { Remaining } 90 \% \\
(N 760 \text {, weighted } N 1147)\end{array}$} & \multirow[b]{2}{*}{$P$ value } \\
\hline & Mean & SD & Mean & SD & & Mean & SD & Mean & SD & \\
\hline TFA (g) & 4.55 & 1.00 & $2 \cdot 22$ & 1.85 & $<0.001$ & 1.93 & 0.59 & 0.95 & 0.46 & $<0.001$ \\
\hline TFA (\%TE) & 1.93 & 0.37 & 1.01 & 0.31 & $<0.001$ & 0.95 & 0.21 & 0.46 & 0.17 & $<0.001$ \\
\hline TFA (\%FE) & 2.00 & 0.37 & 1.07 & 0.31 & $<0.001$ & 1.00 & 0.20 & 0.49 & 0.17 & $<0.001$ \\
\hline Total energy $(\mathrm{kJ} / \mathrm{d})$ & 8870 & 3238 & 8117 & 2435 & $<0.001$ & 7753 & 2326 & 7644 & 2326 & 0.7 \\
\hline Total energy $(\mathrm{kcal} / \mathrm{d})$ & 2120 & 774 & 1940 & 582 & $<0.001$ & 1853 & 556 & 1827 & 556 & 0.7 \\
\hline Food energy $(\mathrm{kJ} / \mathrm{d})$ & 8494 & 3088 & 7531 & 2243 & $<0.001$ & 7372 & 2151 & 7259 & 2171 & 0.7 \\
\hline Food energy $(\mathrm{kcal} / \mathrm{d})$ & 2030 & 738 & 1800 & 536 & $<0.001$ & 1762 & 514 & 1735 & 519 & 0.7 \\
\hline Fat (\%FE) & 39.7 & 6.0 & 34.8 & 5.9 & $<0.001$ & 39.0 & 4.5 & 33.8 & $6 \cdot 1$ & $<0.001$ \\
\hline Saturated fat (\%FE) & $15 \cdot 2$ & 3.5 & $13 \cdot 1$ & 3.0 & $<0.001$ & $16 \cdot 8$ & $3 \cdot 2$ & $12 \cdot 0$ & 2.9 & $<0.001$ \\
\hline NMES (\%FE) & 11.5 & 6.40 & $12 \cdot 9$ & 6.7 & 0.01 & 10.2 & 5.7 & $12 \cdot 2$ & 6.5 & 0.008 \\
\hline $\mathrm{Na}(\mathrm{mg} / 4184 \mathrm{~kJ}(1000 \mathrm{kcal}))$ & 1506 & 334 & 1557 & 361 & 0.09 & 1259 & 401 & 1294 & 331 & 0.5 \\
\hline Vitamin C (mg/4184 kJ (1000 kcal)) & $36 \cdot 3$ & 27.9 & $48 \cdot 3$ & $30 \cdot 3$ & $<0.001$ & 39.5 & 21.9 & 46.9 & 37.1 & 0.02 \\
\hline Vitamin D $(\mu \mathrm{g} / 4184 \mathrm{~kJ}(1000 \mathrm{kcal}))$ & 1.7 & 1.0 & 1.8 & 1.3 & 0.06 & 1.5 & 1.2 & 1.6 & 1.1 & 0.3 \\
\hline Vitamin E (mg/4184 kJ (1000 kcal)) & 4.8 & 1.3 & $5 \cdot 2$ & 1.7 & 0.003 & 4.6 & 1.3 & 5.6 & $2 \cdot 4$ & $<0.001$ \\
\hline $\mathrm{F} \& \mathrm{~V}^{*}(\mathrm{~g} / 4184 \mathrm{~kJ}(1000 \mathrm{kcal}))$ & 88.2 & $68 \cdot 3$ & 121 & 94.0 & $<0.001$ & 144 & 72 & 170 & 115 & 0.008 \\
\hline
\end{tabular}

$\% \mathrm{FE}$, percentage of food energy; \%TE, percentage of total energy; NMES, non-milk extrinsic sugars; F\&V, fruit and vegetables.

${ }^{*}$ Total grams of $F \& V$ do not include pulses, baked beans and fruit juice. 
economic class or education ${ }^{(39)}$; such individuals who were true high consumers of TFA may have been misallocated to the lower TFA groups, potentially masking associations between social disadvantage and high TFA intake. Also disaggregation of composite dishes and allocation of the components to food groups has been more extensive in the NDNS RP than the earlier NDNS surveys; there may be an overestimation of meat and underestimation of fruit and vegetables from composite dishes in the earlier NDNS compared with the later NDNS RP ${ }^{(40)}$.

Weaknesses in comparing social inequalities between pre- and post-reformulation data sets include the lack of comparable IMD data or equivalised household income for the 2000/01 survey. In the post-reformulation survey there were very low numbers ( $n$ 22) consuming above the WHO TFA limit, indicating there may be insufficient power to find differences between TFA intake groups based on the WHO limit; therefore, we suggest that focus should lie on the results of the top $10 \%$ analyses, especially for the recent NDNS RP 2010-2012 ( $n$ 88). Additionally, total energy including alcohol may have diluted the findings in relation to the WHO TFA recommendation. Lower TFA consumers drank more alcohol than high consumers pre-reformulation; higher alcohol consumers may have been more likely to be below the WHO TFA limit because TFA made up a smaller proportion of their total energy. In the UK, individuals of higher socioeconomic class tend to have higher alcohol consumption $^{(41)}$; the association between socio-economic class and TFA intake could be confounded by alcohol intake. Energy from alcohol was not excluded in the calculation to determine the groups above and below $1 \%$ TE from TFA to remain in line with the WHO recommendation. Although energy intake from alcohol is usually a small percentage of total energy, analysis of the top $10 \%$ in terms of food energy intake removed this potential problem.

The main analyses were based on mean intake over the seven or four assessment days; however, these short-term dietary instruments can cause overestimation of the percentage of people in the extremes, due to within-person variation, which can be pronounced for infrequently consumed foods. TFA, however, have been ubiquitous prior to reformulation in processed foods, as well as naturally occurring in meat and dairy products. Daily variability in intakes may still lead to an overestimation of the proportion above the WHO recommended limit on TFA. We used a relatively simple approach to model usual intake using the common framework method with transformed TFA intakes ${ }^{(29)}$, which has some limitations. As expected, this 'usual' intake distribution was narrower than that used for the main analysis, with $37 \%$ instead of the original $57 \%$ estimated to consume over the WHO TFA recommendation in the pre-reformulation survey. This group still showed associations with socio-economic disadvantage. Although the $7 \mathrm{~d}$ data in the prereformulation survey could be converted to $4 \mathrm{~d}$ data to make them more comparable ${ }^{(42)}$, we believe it is preferable to keep as much data as possible and more informative to present these findings for the earlier survey rather than simulated results. In any case, the population means presented should not be significantly affected by whether $4 \mathrm{~d}$ or $7 \mathrm{~d}$ dietary intake has been collected.

The update of TFA values in the NDNS nutrient database appears to be comprehensive for processed foods. However, the TFA values used in these NDNS surveys are an average of a small variety of popular foods and brands from large manufacturers and retailers; this average could mask important TFA differences between foods regularly purchased by different groups in society. For instance, lower income groups may consume budget foods potentially higher in TFA than the average values provided from the composite samples used in the NDNS. Lower income groups may consume products from small and mediumsized enterprises which might be less likely to supply reformulated products; such products are unlikely to be incorporated in the nutrient databanks used to calculate TFA intake in the NDNS. Further research in this area is needed to explore whether low budget or niche brand/ international foods from smaller retailers have higher TFA content than the products underpinning nutrient databanks. TFA values in the food composition databanks underpinning NDNS estimates would be more representative if they were weighted by market share.

The policy context in which UK reformulation efforts occurred is important to determine the drivers of reformulation. Data from other European countries could show whether voluntary reformulation elsewhere has been effective in reducing inequalities in TFA intake. Legislated bans may be preferable where the capacity for routine monitoring of dietary intake and food composition is lower. With the Danish iTFA ban, compliance was evaluated by the government through targeted sampling and analyses of foods after the law was introduced ${ }^{(13)}$. A similar evaluation has not yet taken place in the UK, despite the voluntary Public Health Responsibility Deal being introduced in $2011^{(10)}$. The authors are not aware of any such evaluation of voluntary reformulation in other countries, and the present paper attempted to address this gap. Although voluntary, such iTFA reduction approaches are a distinct policy choice and require rigorous evaluation and monitoring, ideally styled on the 'gold standard' salt reduction initiatives led by the UK Food Standards Agency with government targets, reporting and monitoring ${ }^{(43)}$. Furthermore, caution is needed when extrapolating voluntary iTFA reduction to other policy areas, such as sugar reduction. Table sugar is considered by the industry to have functional properties that are difficult to replace, including taste profile, bulk and texture ${ }^{(44)}$. This makes voluntary sugar reduction potentially more challenging and is being resisted by some UK manufacturers ${ }^{(45)}$. Consequently, there have been calls for alternative approaches for sugar including taxes, portion size controls, restrictions on 
pricing promotions and mandatory limits ${ }^{(46)}$. Despite some technical complexity in reformulating products to remove iTFA, alternatives were available that provided the same functional attributes at minimal additional cost, while providing health benefits ${ }^{(47,48)}$. The shift was also fuelled by potential national or supranational legislation affecting domestic and export markets.

\section{Conclusion}

Voluntary reformulation appeared effective in reducing the TFA content of many UK products. High TFA consumption was associated with socio-economic disadvantage pre-reformulation, but post-reformulation results were less clear regarding inequalities. Similar research should be undertaken in other European countries with voluntary reformulation as well as in countries with legislated bans. Additional research is needed to determine whether products containing high iTFA levels are still sold in the UK and whether these are more likely to be purchased by lower income groups.

\section{Acknowledgements}

Financial support: This work has been funded by the WHO. Co-authors J.J.B. and J.J. are staff members of the WHO Regional Office for Europe. The authors are responsible for the views expressed in this publication and they do not necessarily represent the decisions or stated policy of WHO. Conflict of interest: The University of Leeds has established a spin-out company, myfood24, for its new online dietary assessment tool. J.E.C. is a director and shareholder of myfood24. Authorship: J.J.B., J.J., J.E.C., J.H. and H.L.R. were all involved in the design of the research. J.H. and H.L.R. undertook the analyses and wrote the first draft of the manuscript. All authors reviewed and revised the manuscript. Ethics of human subject participation: Not applicable as this was a secondary analysis of existing data.

\section{References}

1. de Souza RJ, Mente A, Maroleanu A et al. (2015) Intake of saturated and trans unsaturated fatty acids and risk of all cause mortality, cardiovascular disease, and type 2 diabetes: systematic review and meta-analysis of observational studies. BMJ 351, h3978.

2. Mozaffarian D, Katan MB, Ascherio A et al. (2006) Trans fatty acids and cardiovascular disease. New Engl J Med 354, $1601-1613$.

3. Brouwer IA, Wanders AJ \& Katan MB (2010) Effect of animal and industrial trans fatty acids on HDL and LDL cholesterol levels in humans - a quantitative review. PLOS One 5, e9434.

4. Brouwer IA (2016) Effect of Trans-Fatty Acid Intake on Blood Lipids and Lipoproteins: A Systematic Review and Meta-Regression Analysis. Geneva: WHO.
5. Allen K, Pearson-Stuttard J, Hooton W et al. (2015) Potential of trans fats policies to reduce socioeconomic inequalities in mortality from coronary heart disease in England: cost effectiveness modelling study. BMJ 351, h4583.

6. World Health Organization (2004) Global Strategy on Diet, PhysicalActivity and Health. http://apps.who.int/iris/bitstream/10665/43035/1/9241592222_eng.pdf?ua=1 (accessed October 2015).

7. World Health Organization (2013) Global Action Plan for the Prevention and Control of Noncommunicable Diseases 2013-2020. http://www.who.int/nmh/events/ncd_action_ plan/en (accessed April 2016).

8. World Health Organization (2003) Diet, Nutrition and the Prevention of Chronic Diseases. Report of a Joint WHO/ FAO Expert Consultation. WHO Technical Report Series no. 916. http://www.who.int/dietphysicalactivity/publications/ trs916/en/ (accessed November 2015).

9. World Health Organization (2015) Eliminating trans fats in Europe: a policy brief. http://www.euro.who.int/ data/assets/pdf_file/0010/288442/Eliminating-trans-fats-inEurope-A-policy-brief.pdf?ua=1 (accessed April 2016).

10. Department of Health (2014) Public Health Responsibility Deal. F3(a). Non use of artificial trans fat. https://respon sibilitydeal.dh.gov.uk/pledges/pledge/?pl=29 (accessed October 2015).

11. Bundesministers für Gesundheit (2009) Verordnung des Bundesministers für Gesundheit über den Gehalt an transFettsäuren in Lebensmitteln (Trans-Fettsäuren-Verordnung). Bundesgesetz BGBl I Nr. 52/2009. Bundesgesetzblatt für die Republik Österreich 2009, 20 August 2009, Teil II.

12. Ministry of Human Resources (2013) Draft Decree of the Ministry of Human Resources on the Highest Permitted Amount of Trans Fats in Food Products, the Conditions of and Inspections by the Authorities on the Distribution of Food Products Containing Trans Fats and the Rules for Tracking the Population's Consumption of Trans Fats. Budapest: Ministry of Human Resources.

13. Ministry of Food Agriculture and Fisheries of Denmark (2014) Executive Order No. 160 of 11 March 2003 on the Content of Trans Fatty Acids in Oils and Fats. Copenhagen: Ministry of Food, Agriculture and Fisheries of Denmark.

14. Mouratidou T, Livaniou A, Saborido CM et al. (2014) Trans Fatty Acids in Europe: Where Do We Stand? JRC Science and Policy Reports. Luxembourg: Publications Office of the European Union, Institute for Health and Consumer Protection.

15. Pearson-Stuttard J, Critchley J, Capewell S et al. (2015) Quantifying the socio-economic benefits of reducing industrial dietary trans fats: modelling study. PLoS One 10, e0132524.

16. Bajekal M, Scholes S, Love H et al. (2012) Analysing recent socioeconomic trends in coronary heart disease mortality in England, 2000-2007: a population modelling study. PLOS Med 9, e1001237.

17. Nelson M, Erens B, Bates B et al. (2007) Low Income Diet and Nutrition Survey. Volume 2: Food Consumption, Nutrient Intake. http://webarchive.nationalarchives.gov.uk/20110110 172432/http://www.food.gov.uk/multimedia/pdfs/lidnsvol02 (accessed October 2017).

18. Bates B, Lennox A, Prentice A et al. (2014) National Diet and Nutrition Survey: Results from Years 1, 2, 3 and 4 Combined of the Rolling Programme (2008/9 - 2011/12). https://www. gov.uk/government/statistics/national-diet-and-nutritionsurvey-results-from-years-1-to-4-combined-of-the-rollingprogramme-for-2008-and-2009-to-2011-and-2012 (accessed October 2015).

19. Committee on Medical Aspects of Food Policy (1991) Dietary Reference Values (DRVs) for Food Energy and Nutrients for the UK. Report of the Panel on DRVS of the Committee on Medical Aspects of Food Policy (COMA). London: The Stationery Office. 
20. Stender S, Astrup A \& Dyerberg J (2012) A trans European Union difference in the decline in trans fatty acids in popular foods: a market basket investigation. BMJ Open 2, e000859.

21. Downs S, Thow A \& Leeder S (2013) The effectiveness of policies for reducing dietary trans fat: a systematic review of the evidence. Bull World Health Organ 91, 262H-269H.

22. Hendry V, Almíron-Roig E, Monsivais P et al. (2015) Impact of regulatory interventions to reduce intake of artificial trans-fatty acids: a systematic review. Am J Public Health 105, e32-e42.

23. Restrepo BJ \& Rieger M (2016) Trans fat and cardiovascular disease mortality: evidence from bans in restaurants in New York. J Health Econ 45, 176-196.

24. Restrepo BJ \& Rieger M (2016) Denmark's policy on artificial trans fat and cardiovascular disease. Am J Prev Med 50, 69-76.

25. Henderson L, Gregory J, Irving K et al. (2002) National Diet and Nutrition Survey: Adults Aged 19 to 64 Years. vol. 2: Energy, Protein, Carbohydrate, Fat and Alcohol Intake. London: The Stationery Office.

26. Food Standards Agency (2007) Re-estimate of trans fat intake in adults. http://webarchive.nationalarchives.gov.uk/2013022 1113759/http://www.food.gov.uk/multimedia/pdfs/reestimat etransfats.pdf (accessed October 2017).

27. Department Health (2011) Nutrient analysis survey of biscuits, buns, cakes and pastries - Summary report. https://www.gov. uk/government/uploads/system/uploads/attachment_data/ file/216388/dh_129728.pdf (accessed October 2015).

28. Department Health (2013) Nutrient analysis of a range of processed foods with particularreference to trans fatty acids Summary report. https://www.gov.uk/government/uploads/ system/uploads/attachment_data/file/167938/Summary_ Report.pdf (accessed October 2015).

29. Dodd KW, Guenther PM, Freedman LS et al. (2006) Statistical methods for estimating usual intake of nutrients and foods: a review of the theory. J Am Diet Assoc 106, 1640-1650.

30. NatCen Social Research (2015) National Diet and Nutrition Survey Years 1-4, 2008/09-2011/12. [data collection], 7 th ed. UK Data Service, SN: 6533. http://dx.doi.org/ 10.5255/UKDA-SN-6533-6 (accessed June 2015).

31. Office for National Satistics (2005) National Diet and Nutrition Survey: Adults Aged 19 to 64 Years, 2000-2001 [data collection]. UK Data Service, SN: 5140. http://dx.doi.org/ 10.5255/UKDA-SN-5140-1 (accessed September 2015).

32. Tardy A, Morio B, Chardigny J et al. (2011) Ruminant and industrial sources of trans-fat and cardiovascular and diabetic diseases. Nutr Res Rev 24, 111-117.

33. Nestel P (2014) Trans fatty acids: are its cardiovascular risks fully appreciated? Clin Ther 36, 315-321.
34. Office for National Statistics (2009) Understanding patterns of deprivation. Regional Trends 41, 93-114.

35. Livingstone M, Prentice A, Strain J et al. (1990) Accuracy of weighed dietary records in studies of diet and health. BMJ 300, 708-712

36. Rennie KL, Coward A \& Jebb SA (2007) Estimating underreporting of energy intake in dietary surveys using an individualised method. BrJ Nutr 97, 1169-1176.

37. Harper H \& Hallsworth M (2016) Counting Calories - How Under-Reporting Can Explain the Apparent Fall in Calorie Intake. London: Behavioural Insights Team.

38. Lennox A, Bluck L, Page P et al. (2014) Misreporting in the National Diet and Nutrition Survey Rolling Programme (NDNS RP): summary of results and their interpretation. https://www.food.gov.uk/sites/default/files/ndns-appendixx.pdf (accessed April 2017)

39. Poslusna K, Ruprich J, de Vries JH et al. (2009) Misreporting of energy and micronutrient intake estimated by food records and 24 hour recalls, control and adjustment methods in practice. Br J Nutr 101, Suppl. 2, S73-S85.

40. Fitt E, Mak TN, Stephen AM et al. (2010) Disaggregating composite food codes in the UK National Diet and Nutrition Survey food composition databank. Eur J Clin Nutr 64, Suppl. 3, S32-S36.

41. Craig R \& Mindell J (2013) Health Survey for England 2012. London: The Health and Social Care Information Centre.

42. Pot GK, Prynne CJ, Roberts C et al. (2011) National Diet and Nutrition Survey: fat and fatty acid intake from the first year of the rolling programme and comparison with previous surveys. Br J Nutr 107, 405-415.

43. Food Standards Agency (2013) UK Salt Reduction Initiatives. London: FSA.

44. Struck S, Jaros D, Brennan CS et al. (2014) Sugar replacement in sweetened bakery goods. Int J Food Sci Technol 49, 1963-1976.

45. Smyth C (2017) Food giants reject lower sugar target. The Times, 22 March 2017. https://www.thetimes.co.uk/article/ food-giants-reject-lower-sugar-target-s027v29t5 (accessed October 2017).

46. Tedstone A, Targett V \& Allen R (2015) Sugar Reduction: The Evidence for Action. London: Public Health England.

47. Eckel RH, Borra S, Lichtenstein AH et al. (2007) Understanding the complexity of trans fatty acid reduction in the American diet. Circulation 115, 2231-2246.

48. Flickinger BD \& Huth PJ (2004) Dietary fats and oils: technologies for improving cardiovascular health. Curr Atheroscler Rep 6, 468-476. 